\title{
3 Umwelt in Gesellschaft, Politik und Recht
}

\subsection{Verantwortungsethik für Umwelt und Natur}

Wolfgang Liebert

Institut für Sicherheits- und Risikowissenschaften,

Department für Wasser-Atmosphäre-Umwelt (WAU)

liebert@boku.ac.at

\subsubsection{Hintergrund}

Die Umweltdebatte hat in den westlichen Industriestaaten erst seit den 1960er- und 1970er-Jahren ernsthaft Fuß fassen können. Dafür gab es mehrere Auslöser: ein wachsendes Umweltbewusstsein infolge größerer technischer Unfälle, ein schleichender zunächst teilweise kaum sichtbarer - Prozess der ökologischen Degradation, Gesundheitsgefährdungen sowie die Entstehung global wirksamer Problemlagen durch technisches Handeln. Die Diskussionen der Umweltbewegung und -politik erreichten auch die Philosophie. Seit den 1970er-Jahren entwickelte sich eine neue Bereichsethik, die Umweltethik. Sie baut auf traditionellen ethischen Konzeptionen auf und fragt nach ethisch-moralischer Orientierung für das menschliche Umwelthandeln.

Stellvertretend dafür stellt dieser Beitrag die Ethikkonzeption von Immanuel Kant sowie die Verantwortungs- und Zukunftsethik von Hans Jonas in Hinblick auf die Entwicklung umweltethischer Ansätze vor. Abschließend folgt ein knapper Ausblick auf die Breite der umweltethischen Debatte.

\subsubsection{Kants Maximenethik: Oberste Vernunftprinzipien der Moral}

Wie kann unsere innere moralische Stimme gut zum Ausdruck kommen? Wie können wir uns selbst leiten? Wie kann etwas für alle Einsichtiges und (irgendwie) Verbindliches (und damit vielleicht Verpflichtendes) ausfindig gemacht werden, ohne in moralischen Dogmatismus ${ }^{1}$ oder den Relativismus der Beliebigkeit in Moralfragen zu verfallen?

Immanuel Kant (1724-1804) hat in seinen ethischen Schriften versucht, Antworten zu geben. Er ging dabei ganz bewusst nicht von Gefühlen aus, sondern bezog sich auf das Vernunftwesen Mensch. Die Fähigkeit zum eigenständigen Nachdenken ist eine menschliche Gabe und wird zur Aufgabe für das Gelingen einer freiheitlichen menschlichen Zivilisation. Oberste moralische Prinzipien sollen allein aus Vernunft-

1 Hier im Sinne des Vertretens von festgefügten ethischen Lehrmeinungen oder Normen in einer Weise, die keine Kritik daran zulässt. 


\section{Umwelt in Gesellschaft, Politik \& Recht}

gründen einsehbar und gültig sein - ohne (direkten) Bezug auf Erfahrungen in der Welt (Empirie). Sie sollen vor aller Erfahrung (a priori) gelten. Kant will die Moral tief in der Vernunft des Menschen verankern - unabhängig von rein empirischen, von Fall zu Fall wechselnden Bedingungen des menschlichen Handelns.

Der erste Satz seiner Grundlegung der Metaphysik der Sitten ${ }^{2}$ lautet: „Es ist ... nichts in der Welt, was ohne Einschränkung für gut könnte gehalten werden, als allein ein guter Wille“ (Kant 1785/1975, BA S. 1). Der Gehalt dieses Satzes meint mindestens zweierlei: Erstens ist die normative Idee eines uneingeschränkt Guten grundlegend für die Sittlichkeit (Moralität) einer Person und für das Zusammenleben der Menschen. Zweitens wird die Willensfähigkeit als besondere Eigenschaft des Vernunftwesens Mensch hervorgehoben. Das, was den Menschen auszeichnet, ist also nicht nur seine Vernunft allein, sondern auch sein freier Wille. Wenn das so ist, muss jeder Mensch sein willentliches Handeln genauer ansehen und prüfen, ob es dem Guten dient.

Gibt es ein objektives Kriterium für Moralität, das a priori Gültigkeit beanspruchen kann? Kann man bestimmen, welche grundlegenden Handlungsprinzipien oder Handlungsmaximen moralisch vertretbar sind? Gemäß Kant sind dies Appelle an die menschliche Pflicht, die in Form von Imperativen formuliert werden (,handle so und so"). Die Verwendung des Pflichtbegriffs durch Kant mag heute irritieren, aber dies erfolgte im Geiste der Aufklärung. Es handelt sich also nicht um Befehle, die dem einzelnen Menschen von äußeren Mächten, von überlegenen machtvollen Autoritäten auferlegt werden, sondern es geht um die Selbstgesetzgebung des vernünftigen, freien und autonomen Menschen.

Der gesuchte Typ des Imperativs ist für Kant ein kategorischer Imperativ. Vereinfacht ist damit gemeint: Es soll etwas formulierbar werden, das über alle Funktionalisierungen und rein subjektive Absichten des Handelns hinausreicht und Anspruch auf voraussetzungslose, eben „kategorische“ Verbindlichkeit erheben kann. Gesucht ist ein Imperativ der allgemeinst denkbaren Form mit universeller Gültigkeit und Brauchbarkeit, der für jeden vernunftbegabten Menschen einsichtig und anwendbar ist.

Kants kategorischer Imperativ in seiner ersten Formulierung lautet: „Handle nur nach derjenigen Maxime, durch die du zugleich wollen kannst, dass sie ein allgemeines Gesetz werde" (Kant 1785/1975, BA S. 52).

Wie „funktioniert" der kategorische Imperativ? Eine Maxime ist eine praktische Regel, d.h. eine Regel für das Handeln, welche durch die Vernunft bestimmt wird. Eine Maxime ist also ein überlegter Grundsatz (oder das Prinzip), nach dem das Subjekt handeln will. Der kategorische Imperativ - als vernünftiger, objektiver, allgemeingülti-

2 Zitiert wird nach Seiten der Originalausgabe, wie üblich mit BA für die erste (A) und die zweite Auflage (B), sodass die Stellen in jeder guten Kant-Ausgabe aufgefunden werden können. 
ger Prüfalgorithmus - soll nun eine Überprüfung der moralischen Vertretbarkeit von subjektiven Handlungsregeln ermöglichen: Steckt hinter der Maxime des Handelns, die das Subjekt sich (probehalber) setzt, wirklich ein guter Wille? Nur wenn ich wollen kann, dass meine Maxime des Handelns auch allgemeines Gesetz werden könnte, nur dann ist meine Maxime moralisch vertretbar. Das objektive Prinzip (der kategorische Imperativ, das ,praktische Gesetz“) ist gültig für jedes vernünftige Wesen und ist der Grundsatz, nach dem es letztlich sein Handeln ausrichten soll (Kant $1785 / 1975$, BA S. 52). So ist zu unterscheiden zwischen einer objektiven, allgemeingültigen Ebene (das auf das praktische Handeln bezogene Gesetz, der kategorische Imperativ) und der subjektiven Ebene (die Maximen, die sich die Individuen setzen).

Die Maximen sind nicht von außen vorgeschrieben. Sie dienen der vernünftigen Selbstbestimmung des Subjekts. Ganz im Geiste der Aufklärung werden damit auch angemaßte Autoritäten, autoritär auftretende Staatsführungen oder Kirchen radikal infrage gestellt. Der kategorische Imperativ sorgt dafür, dass nicht irgendwelche selbst gesetzten Maximen als moralische Orientierung genutzt werden. Er zielt auf die Überprüfung der moralischen Vertretbarkeit von Maximen ab. Was ich (das Subjekt) tun soll, folgt meiner eigenen Einsicht; es sind zunächst einmal Ansprüche von mir an mich selbst. An das als richtig Anerkannte soll ich mich dann auch halten. Sie betreffen die Lebensführung insgesamt.

Was Kant entwickelt hat, stellt sehr hohe Ansprüche an jeden einzelnen Menschen. Jede und jeder muss sich über mögliche Maximen des eigenen Handelns klar werden und dann prüfen, ob diese moralisch vertretbar sind, d.h. alle anderen zunächst probehalber angenommenen, möglichen Maximen wieder aussondern. Die als moralisch vertretbar erkannten Maximen sollen schließlich auch befolgt werden. Betont sei: Der kategorische Imperativ ist kein Prüfkriterium für Einzelhandlungen und auch keine Anleitung für ein Gesetzgebungsverfahren.

Der Test auf Verallgemeinerbarkeit von zunächst persönlichen Maximen öffnet die personale, individuelle Perspektive hin zur intersubjektiven Perspektive des Menschen als Gemeinschaftswesen. Der kategorische Imperativ als Prüfkriterium soll tendenziell auch dafür sorgen, dass die jeweiligen subjektiven Lebenshorizonte, die mit Maximen verknüpft werden, auch in intersubjektive Lebenshorizonte von menschlichen Gemeinschaften passen.

Die dritte Formulierung des kategorischen Imperativs ${ }^{3}$ lautet: „Handle so, dass du die Menschheit, sowohl in deiner Person, als in der Person eines jeden anderen, jederzeit zugleich als Zweck, niemals bloß als Mittel brauchst“" (Kant 1785/1975, BA S. 66f.). Kants Erläuterungen dazu sprechen für sich: „der Mensch ist keine Sache“; der

${ }^{3}$ Es gibt noch eine zweite und vierte Formulierung, auf die hier nicht näher eingegangen wird. 


\section{Umwelt in Gesellschaft, Politik \& Recht}

Mensch ist „nicht etwas, das bloß als Mittel gebraucht werden kann“; der Mensch „muss bei allen seinen Handlungen jederzeit als Zweck an sich selbst betrachtet werden“ (Kant 1785/1975, BA S. 66f.). Dies ist gleichbedeutend mit der „Autonomie und Würde ... der menschlichen und jeder vernünftigen Natur" (Kant 1785/1975, BA S. 79).

Der Philosoph Ernst Tugendhat hat die dritte Formulierung des kategorischen Imperativs knapp zusammengefasst: „Instrumentalisiere niemanden“ (Tugendhat 1993, S. 80). Man könnte vielleicht auch sagen: Jeder Mensch hat Würde und Eigenwert und darf nicht verzweckt werden. Dies zeigt sehr deutlich, dass Kant nicht bei einer rein subjektivistischen, nur für das Individuum selbst gültigen moralischen Position stehen bleibt, auch wenn er eine personale Formulierung wählt. Hier steht ein allgemein verbindlich gemeintes „Prinzip der Menschheit“ (Kant 1785/1975, BA S. 69), ein Menschheitsgebot oder Menschheitsgesetz. Hier ist das angesprochen, was wir heute Menschenwürde nennen.

Kants moraltheoretische Überlegungen sind für den zwischenmenschlichen Bereich entwickelt worden. Sie könnten aber möglicherweise auch eine bewusstere Reflexion menschlichen Handelns mit Relevanz für die Naturzusammenhänge, in die wir eingebunden sind, anleiten. Ich könnte überlegen, ob es in meinem alltäglichen Handeln Handlungsmaximen gibt, die Umweltfolgen in vernünftiger Weise im Blick haben. Wenn ich mögliche Maximen bzw. grundlegende Handlungsprinzipien erkenne, könnte ich den kategorischen Imperativ anwenden, um zu prüfen, ob sie moralisch vertretbar sind und ob sie - zumindest tendenziell - allgemeingültig sein könnten (Könnten sie allgemeines Gesetz werden? Könnte ich das wollen?). So könnte die bewusste Berücksichtigung von Umweltfolgen oder allgemeiner von Folgen des Handelns

\section{Fallbeispiel 3.1.1: Reduktion des persönlichen $\mathrm{CO}_{2}$-Beitrags}

Bekanntlich ist der $\mathrm{CO}_{2}$-Ausstoß von technischen Prozessen, die Menschen nutzen, eine wesentliche Ursache für den stattfindenden Klimawandel. Ich könnte nach einer Handlungsmaxime suchen, die meinen $\mathrm{CO}_{2}$-Beitrag auf ein vertretbares Maß reduzieren kann. Welche Jahresmenge an $\mathrm{CO}_{2}-$ verursacht durch die ganze Menschheit - gilt nach wissenschaftlicher Erkenntnis noch als vertretbar? Diese Zahl dividiere ich durch die Gesamtzahl der Menschen und erhalte wohl etwa 2-3 Tonnen pro Jahr. Ich stelle als Handlungsmaxime auf, dass ich im Jahresmittel durch mein Handeln (Stromverbrauch, Wärmebedarf, Mobilität, Kleidung, Nahrung, Herstellung und Nutzung technischer Geräte, Infrastruktur in meiner Lebensregion etc.) nur die noch vertretbare $\mathrm{CO}_{2}$-Menge der Weltbevölkerung pro Kopf freisetzen soll. Zur Umsetzung müsste ich herausfinden, wie viel $\mathrm{CO}_{2}$ meine alltäglichen Verrichtungen und genutzten Güter freisetzen, und dann der Maxime entsprechende Verbrauchsreduktionen vornehmen, um meine $\mathrm{CO}_{2}$-Freisetzung auf das notwendige Maß (wohl etwa auf die Hälfte oder ein Drittel) zu reduzieren. Es ist nun zu überlegen: Wäre das vernünftig? Kann ich das als Individuum erreichen? Kann dies ohne weitere gesamtgesellschaftliche Maßnahmen gelingen? Hält die gewählte Maxime der Überprüfung durch den kategorischen Imperativ stand? Wäre eine solche ProKopf-Regel für alle Menschen in allen Erdregionen sinnvoll, fair und akzeptabel? 
in Naturzusammenhängen als mit zu berücksichtigendes Handlungsmotiv in die Beurteilung von Handlungsmaximen eingehen - zumindest auf der subjektbezogenen Ebene. Offen oder unklar bliebe allerdings, wie wir zu allgemeingültigen ethischen Forderungen oder gar zu konkreten Gesetzen kommen können, die auf allgemein akzeptierten moralischen Überlegungen basieren.

Die dritte Formulierung des Kategorischen Imperativs als Menschheitsgesetz könnte in den Bereichen zum Zuge kommen, in denen wir den Eindruck haben müssen, dass die Menschenwürde bei umweltrelevantem Handeln verletzt wird (z.B. Einbringung umweltund gesundheitsgefährdender Stoffe in die Natur, exzessive Mineraliengewinnung durch Menschen und die Natur gefährdende Abbauprozeduren wie bei größeren, offenbar billigend in Kauf genommenen Minenunglücken in Brasilien 2015 und 2019 erlebt).

\subsubsection{Verantwortungsethik und neuer Imperativ (Hans Jonas)}

Hans Jonas (1903-1993) hat 1979, als über 75-Jähriger, ein Buch vorgelegt, das in der Debatte über die ökologische Krise Furore machte: „Das Prinzip Verantwortung Versuch einer Ethik für die technologische Zivilisation“. Jonas glaubte nicht, dass die traditionelle Ethik mit den Herausforderungen in der wissenschaftlich-technischen Welt, in der wir leben, angemessen umgehen kann. Kennzeichen der neuen Situation (Jonas 1979, S. 15ff.) sind: die „beispielslose Reichweite“ menschlicher Handlungen „in die Zukunft“, die „räumliche Ausbreitung und Zeitlänge der Kausalreihen“ über die „Nahsphäre hinaus“, der „irreversible“ Charakter von Veränderungen sowie eine „kritische Verletzlichkeit der Natur durch die technische Intervention des Menschen“bis an die Toleranzgrenzen der Natur. Die Menschheit tritt als „kollektiver Täter“ auf, und ,individuelle Taten“ sind nicht mehr wirklich zurechenbar (Jonas 1979, S. 32). Dies ist eines der zentralen Argumente, warum die traditionelle, auf das individuelle Tun abzielende Ethik laut Jonas nicht mehr zureichend ist.

Als Konsequenz wird eine neue Moralität eingefordert: „Neuartige Vermögen des Handelns [erfordern] neue Regeln der Ethik und vielleicht sogar eine neuartige Ethik“" (Jonas 1979, S. 58). Den Kern einer neuen Ethik soll ein „neuer Imperativ“ bilden. Bei Kant wurde der kategorische Imperativ noch „zur Probe ... private[r] Wahl“, so Jonas (1979, S. 57). Nun müsse es aber um weit mehr gehen. Wir dürften zwar unser eigenes Leben wagen, nicht aber das der Menschheit. Es geht nun um „Handlungen des kollektiven Ganzen“ - bzw. um Handlungen, die das kollektive Ganze betreffen.

Jonas (1979, S. 36) stellt seinen neuen Imperativ ähnlich wie Kant in mehreren Alternativformulierungen auf:

- „Handle so, dass die Wirkungen deiner Handlung verträglich sind mit der Permanenz echten menschlichen Lebens auf Erden; 


\section{Umwelt in Gesellschaft, Politik \& Recht}

oder negativ ausgedrückt:

- Handle so, dass die Wirkungen deiner Handlung nicht zerstörerisch sind für die zukünftige Möglichkeit solchen Lebens;

oder einfach:

- Gefährde nicht die Bedingungen für den indefiniten Fortbestand der Menschheit auf Erden;

oder, wieder positiv gewendet:

- Schließe in deine eigene Wahl die zukünftige Integrität des Menschen als MitGegenstand deines Wollens ein."

Dieser neue Imperativ, den man auch den ökologischen Imperativ nennen könnte, scheint einfach und einleuchtend formuliert zu sein, dennoch ist eine Interpretation nötig. In der ersten Formulierung geht es um die Beachtung der Kausalketten, in die unser (technisch verstärktes) Handeln eingebunden ist. Was sind die angestrebten Wirkungen und was die mitbewirkten unerwünschten Folgen? Und sind diese Wirkungen und Folgen verträglich mit dem dauerhaften Fortbestand menschlichen Lebens? Die Verträglichkeitsforderung wird in der zweiten Formulierung grob skizziert: Handlungen sollen jedenfalls nicht zerstörerisch für „die Permanenz echten menschlichen Lebens“ sein. Was mit „echtem“ menschlichem Leben gemeint sein könnte, kann man zunächst nur erahnen. Die dritte Formulierung verlangt im Grunde, dass geklärt wird, was die Grundbedingungen zur Erreichung des Fortbestandes der Menschheit sind. Und es wird klar, dass bewusst eine anthropozentrische Haltung eingenommen wird. Es scheint nicht um die Natur insgesamt zu gehen, sondern der Maßstab ist das Überleben der Menschheit und das „echte“ menschliche Leben. Die vierte Formulierung legt nahe, vor der Durchführung jeder Handlung, die ja willensbasiert ist und zumeist eine Wahl zwischen Alternativen darstellt, zusätzliche Überlegungen anzustellen: Die „zukünftige Integrität des Menschen“ ist genauso bedeutsam wie der konkrete erwartete Ertrag aus der Handlung. Die „Integrität“ des Menschen spielt wieder auf die anfängliche Formulierung des „echten“ menschlichen Lebens an. Es geht also nicht nur um die pure Überlebenssicherung für ein irgendwie geartetes menschliches Leben auf diesem Planeten.

In einem weiteren Text von Jonas wird sehr deutlich, dass er die anthropozentrische Grundhaltung durchaus kritisch reflektiert. Schon im „verständigen Selbstinteresse“ des Menschen sei es, eine „Durchbrechung der Anthropozentrik“ anzugehen. „Als planetarische Macht ersten Ranges darf er nicht mehr nur an sich selbst denken“ (Jonas 1993, S. 85). Eine „Umweltethik“ werde nötig, denn jetzt beanspruche „die gesamte Biosphäre des Planeten mit all ihrer Fülle von Arten, in ihrer neu enthüllten 
Verletzlichkeit gegenüber den exzessiven Eingriffen des Menschen ihren Anteil an der Achtung, die allem gebührt, das seinen Zweck in sich selbst trägt - d.h. allem Lebendigen" (Jonas 1993, S. 85). Bemerkenswert ist, dass Kants dritte Formulierung des kategorischen Imperativs, die den Eigenwert jedes Menschen betont, hier erweitert wird auf nichtmenschliches Leben auf diesem Planeten.

Wie kann man nun unter Nutzung des neuen Imperativs konkret vorgehen? Aus Jonas' Sicht besteht die Schwierigkeit dabei darin, dass das konkrete Folgewissen über technisches Tun in der Welt hinter dem technischen Wissen her hinkt. ${ }^{4}$ Was kann man dennoch ethisch verantwortlich tun? Wie kann eine "Zukunftsethik“ (Jonas 1979, S. 39) praktisch aussehen? Jonas schlägt zwei wichtige Elemente der Vorgehensweise einer Zukunftsethik vor: eine „Heuristik der Furcht" und einen „Vorrang der schlechten Prognose“.

„Heuristik der Furcht“ (Jonas 1979, S. 63ff.) meint, dass die Furcht als Erkenntnis gewinnendes Instrumentarium genutzt werden soll. Wir müssten erstens eine Vorstellung von den Fernwirkungen unseres technischen Handelns in der Welt gewinnen und eine Haltung dazu einnehmen. Man müsse sich auf das Fürchten einlassen und das mögliche Schlechte, das bewirkt werden kann, als etwas bereits jetzt Erfahrbares erkennen. Zweitens müsse man dann ein angemessenes Gefühl entstehen lassen (entsprechend dem Vorgestellten). Man müsse „sich vom erst [nur] gedachten Heil und Unheil kommender Geschlechter affizieren ... lassen“. Dies soll eine „Furcht geistiger Art“ sein, jedoch nicht eine Furcht, die uns von außen befällt und ohnmächtig macht. Sie soll helfen, eine Haltung zu den Dingen einzunehmen, die durch das kollektive Handeln der Menschheit kommen können.

Damit Nutzenerwägungen die Schadensbedenken nicht überschatten oder übertrumpfen können, steht der mögliche Nutzen aus menschlichem Handeln nicht im Vordergrund. Die Furcht vor Schädigungen, auf die man sich einlassen soll, muss allerdings eine „begründete Furcht“ sein (Jonas 1979, S. 392). Sie soll nicht rein gefühlsmäßig bleiben, sondern muss auch für andere nachvollziehbar begründet werden können.

Als zweites Element der Vorgehensweise schlägt Jonas (1979, S. 70ff.) den „Vorrang der schlechten vor der guten Prognose" vor. Angesichts der Unsicherheit aller Zukunftsprojektionen und dem Vorwärtsdrängen des „Großunternehmens der modernen Technologie“ als Ganzem müsse „die Vorschrift, primitiv gesagt, [heißen,] dass

4 Für Jonas war wohl die in den 1970er-Jahren neu entstehende Technikfolgenabschätzung noch kein überzeugendes Konzept, mit dem diese Wissenslücke überbrückt werden könnte. Heute gibt es Bemühungen zur vorausschauenden, prospektiven Wissenschafts- und Technikfolgenforschung (Liebert und Schmidt 2010, 2018). Weiterhin gibt es viele historische Beispiele, in denen durchaus frühzeitige Warnungen ausgesprochen wurden. Lehren wurden aber erst viel später daraus gezogen. Umweltrelevante Beispiele finden sich in UBA (2004). 


\section{Umwelt in Gesellschaft, Politik \& Recht}

der Unheilsprophezeiung mehr Gehör zu geben ist als der Heilsprophezeiung". Wesentlich ist hier, dass Jonas das Fortschreiten des kollektiven technologischen Entwickelns und Tuns als Großunternehmen sieht, das wie eine große Dampfwalze voran rollt. Eine evolutive Kleinschrittigkeit, wie sie in den Naturprozessen als natürliche Entwicklung beobachtbar ist, sei hier nicht (mehr) gegeben. Der „Vorteil der tastenden Natur“ werde ausgehebelt. Der Evolution sei es fremd, aufs Ganze zu gehen. Die Menschheit gehe aber aufs Ganze.

Jonas verwendet die mit der Anmutung der Irrationalität verbundenen Begrifflichkeiten der Heils- und der Unheilsprophezeiung. Zukünftiges Unheil durch Technik zu befürchten, erscheint ebenso rational bzw. irrational wie das zukünftige Heil durch Technologie zu versprechen. Jonas ist überzeugt, dass eine Unheilsprognose essenziell bedeutsamer für die Zukunft der Menschheit sein könne. Diese Vorrangregel hat deutliche Kritik hervorgerufen, weil sie als Innovationsverhinderung interpretiert wurde. Umgekehrt ist sie der Keim des Vorsorgeprinzips, das inzwischen in Europa einen hohen Stellenwert hat. Das Vorsorgeprinzip ist nicht auf individuelles Verhalten beschränkt, sondern nimmt kollektives menschliches Tun in den Blick.

Die zwei Elemente der Vorgehensweise werden ergänzt durch das Verantwortungsgefühl und die tatsächliche Übernahme von Verantwortung: „Erst das hinzutretende Gefühl der Verantwortung, welches dieses Subjekt an dieses Objekt bindet, wird uns seinethalben handeln lassen" (Jonas 1979, S. 170). Damit erkennt Jonas ein wesentliches affektives oder emotionales Element der Ethik an. Erst das in uns entstehende Verantwortungsgefühl lässt uns verantwortlich, moralisch handeln. Die Vernunft allein, die rationale Reflexion allein kann dies nicht bewirken. Die „Sorge um den Nachwuchs“ (Jonas 1979, S. 85ff.) ist „der elementar menschliche Urtyp des Zusammenfalls von objektiver Verantwortlichkeit und subjektivem Verantwortungsgefühl“ (Jonas 1979, S. 171). Dies kennen wir als menschliche Wesen, wenn wir beobachten, wie Mütter und Väter auf Babys und Kleinkinder einfühlend und sorgsam reagieren. Je kleiner die jungen Wesen sind, desto stärker werden in der Regel die Menschen von dieser zuneigenden fürsorglichen Haltung berührt und selbst erfasst. Jonas bemerkt, dass diese Sorge um den Nachwuchs der Anrufung des Sittengesetzes à la Kant gar nicht bedürfe.

Jonas zielt damit auf ein nichtreziprokes Verantwortungsverhältnis ab, das nicht (unbedingt) auf erwarteter Gegenseitigkeit beruht. Es besteht für den Handelnden jenseits von Gerechtigkeitsvorstellungen oder Nutzenerwartungen. Diese Art von Verantwortung besteht objektiv. Das heißt, man kann von ihr auch nicht zurücktreten (so wie man ein übernommenes Amt zurücklegen kann), sondern sie ist einfach vorhanden und global wirksam. Sie ist „unbedingt und unwiderruflich“ (Jonas 1979, S. 173). Anthropozentrisch formuliert: „Das Urbild aller Verantwortung ist die von Menschen für Menschen“ (Jonas 1979, S. 184). Man kann jedoch ergänzen, dass die Sorge um den 
Nachwuchs auch im Tierreich beobachtet werden kann und dass die Verantwortung nicht auf den Menschen beschränkt bleiben muss. Das Verantwortungsverhältnis kann im Prinzip für alles Lebendige ,in seiner Bedürftigkeit und Bedrohtheit“ (Jonas 1979, S. 185) gelten. Für Jonas kann nur Lebendiges Gegenstand von Verantwortung werden.

Schließlich betont Jonas, dass es den Menschen auszeichne, Verantwortung haben zu können. Der Mensch ist nicht nur ein vernunftbegabtes Wesen (animal rationale), sondern ein „moralisches Wesen“" (Jonas 1979, S. 185). Jonas spricht konkret von der „Höchste[n] Pflicht der Bewahrung“ (Jonas 1979, S. 74) und von der „Hütung des Erbes“, der „Integrität des Ebenbildes“ (Jonas 1979, S. 393), womit der Religionsphilosoph Jonas offenbar auf die biblische Gottesebenbildlichkeit des Menschen an-

\section{Fallbeispiel 3.1.2: Umgang mit Gene-Drive-Forschung}

Bei der Übertragung von gefährlichen Krankheiten wie Malaria, die jedes Jahr sehr viele Opfer fordern, spielen bestimmte Mückenarten eine zentrale Rolle. Fruchtfliegen (Drosophila) verschiedener Arten können Ernteerträge (z.B. von Kirschen oder Oliven) erheblich dezimieren. Gentechnisch erzeugte Veränderungen der Insekten werden als Gegenmittel erforscht - verstärkt seit der Entdeckung der neuartigen Genschere CRISPR/Cas im Jahr 2012. Es wird versucht, Insektenarten gentechnisch so zu verändern, dass sich bestimmte gewünschte Eigenschaften nicht mehr gemäß den Mendel'schen Vererbungsregeln mit 50\% Wahrscheinlichkeit, sondern dominant vererben lassen, sodass rasch ganze Populationen verändert werden. Man spricht von mutagener Kettenreaktion oder Gene Drive. Forschende arbeiten an Eliminierungsprogrammen (z.B. durch Erzeugung von Unfruchtbarkeit) oder an der Verminderung der Pathogenität von Krankheitsüberträgern (z.B. durch Beeinflussung der WirtPathogen-Wechselwirkung). Die Freisetzung von entsprechend gentechnisch veränderten Insekten müsste allerdings jenseits der Laborgrenzen, im Freiland - also in der ganzen Natur - erfolgen. Es gibt Befürchtungen, dass solche Freisetzungen neben erhofften Wirkungen auch erhebliche unerwünschte oder gefährliche Folgen haben könnten. Ein einzelnes Insekt, das mit einem effektiv wirksamen Gene-Drive-Mechanismus ausgestattet ist, könnte die Natur tiefgreifend - vielleicht irreversibel - verändern. Es stellen sich folgende Fragen: Ist die Weitergabe eines durch Gene Drive ausgelösten gentechnischen Programms (auch eines Eliminationsprogramms) über Artgrenzen hinweg möglich? Welche „ökosystemaren" (also ganze Ökosysteme betreffende) Folgen (z.B. durch den Ausfall bestimmter Spezies) sind denkbar? Sind Kaskadeneffekte mit Auswirkungen auf andere Lebewesen möglich? Kann man sicherstellen, dass Veränderungen nur an den angezielten Stellen im Genom vorgenommen werden? Sind unerwartete Effekte möglich? Könnten Infektionserreger den Wirtsorganismus wechseln bzw. andere Fruchtschädlinge den Platz der bekämpften Fruchtfliegen übernehmen, die dann ebenfalls mit Gene Drives bekämpft werden müssten, sodass es zu einer Kettenreaktion mutagener Kettenreaktionen kommen muss? Wären mit Gene Drives veränderte Insekten nicht selfish genetic elements, die quasi autonom in der Natur operieren, was die Kontrolle durch den Menschen infrage stellt? Würde die Eingriffstiefe menschlicher Technik in Naturprozesse nicht ganz erheblich erhöht, bis hin zu der Vision einer Natur unter Menschenhand? Angesichts dieser Risiken muss weiter gefragt werden: Können der neue Imperativ von Jonas, seine Heuristik der Furcht und seine Vorrangregel Anleitung für einen verantwortbaren Umgang mit der Gene-Drive-Forschung geben? Wie kann das Vorsorgeprinzip konkret wirksam werden? Sind Forschungs- und Entwicklungsarbeiten in diesen Bereichen überhaupt verantwortbar? 


\section{Umwelt in Gesellschaft, Politik \& Recht}

spielt, allerdings ohne damit positive Religion als Zugang zur Verantwortungs- und Zukunftsethik einfordern zu wollen. Jonas' konservativ-bewahrende, also tutoristische Position ist erkennbar: Der Mensch ist schon gut, so wie er ist - jenseits aller politischen oder auch technischen Utopie, was die zukünftige (auch technisch inszenierte) Entwicklung von Mensch und Gesellschaft anbetreffen könnte. Dies verdeutlicht wohl auch, was mit der "Permanenz echten menschlichen Lebens" gemeint sein könnte, von dem in Jonas' Imperativ die Rede ist.

\subsubsection{Auf dem Weg zu einer Umweltethik}

Jonas' Verantwortungs- und Zukunftsethik ist so entwickelt worden, dass sie Anleitungen für den menschlichen Umgang mit Umwelt und Natur geben könnte. Dieser Beitrag beschreibt nur zwei mögliche ethische Ansätze auf dem Weg zu einer Umweltethik. Die über 2000 Jahre alte Ethiktradition hat auch andere Konzeptionen hervorgebracht, die für umweltethische Überlegungen fruchtbar gemacht werden können. ${ }^{5}$ Dazu gehören die Tugendethik, der Utilitarismus und gewiss auch religiöse Traditionen. Die Konzeption von Kant wurde in der Diskursethik grundsätzlich so weiterentwickelt, dass die Allgemeingültigkeit von Normen von uns allen gemeinsam überprüfbar erscheint. Der Hoffnung auf eine universelle Vernunftorientierung in ethischen Belangen - jenseits von Dogmatismus und unverbindlichem Relativismus - wird so wieder Leben eingehaucht. Und doch muss auch geklärt werden, welche Rolle Gefühle, die auch von Jonas angesprochen wurden, für die Moral und für die tatsächliche Übernahme von Verantwortung spielen. Ist es nicht so, dass es gar nicht möglich scheint, ein Wertbewusstsein zu entwickeln, ohne (moralisch gefärbte) Gefühle zuzulassen? Kann jemand, der die Stimme eines moralischen Gefühls in sich nicht zulässt, überhaupt Werthaltungen und eine moralische Position - auch gegenüber anderen und der nichtmenschlichen Mitwelt - entwickeln? Was motiviert zum Handeln auf der Basis des als richtig Erkannten?

Für die Umweltethik ist darüber hinaus von großer Bedeutung, wie sie sich zum Anthropozentrismus stellt. Die Frage ist, ob sie sich ganz im Gegensatz zu menschenzentrierten Moralkonzeptionen sehen kann oder ob sie durch gezielte Differenzierungen (z.B. schwacher versus starker Anthropozentrismus) den Herausforderungen durch die Umweltprobleme gerecht werden kann. Es kann auch gefragt werden, ob nicht für eine Aufweitung der Moralgemeinschaft über den nichtmenschlichen Bereich hinaus argumentiert werden kann oder muss. So wird im sogenannten Sentientismus auch allen empfindungsfähigen nichtmenschlichen Wesen moralisch zu respektierender Eigenwert zuerkannt. Ökozentrische Positionen weisen sogar Ökosystemen einen

5 Vergleiche dazu beispielsweise Liebert (2019). 
Eigenwert zu. Spezifischer sind Konzepte einer holistisch angelegten Tiefenökologie oder einer lebenszentrierten Ethik entwickelt worden. Unabhängig und in Abgrenzung von Eigenwertdebatten sind auch - beispielsweise in den USA - pragmatische umweltethische Positionen entstanden, die den Anspruch haben, in konkreten Situationen umweltpolitische Entscheidungsprozesse (ganz pragmatisch) durch ethische Argumentation (weniger theorielastig) zu unterstützen. Für die Entwicklung einer Umweltethik und zur Erreichung einer umweltethischen Orientierung ist es von Bedeutung, die Breite der möglichen Konzeptionen in ihren Grundzügen zu verstehen, ihren jeweiligen Gehalt zu sichten und zu interpretieren (Liebert 2019; Ott et al. 2016).

Wir stehen gemeinsam vor der Frage, wie wir uns gegenüber der Natur, auf deren Basis wir leben und uns weiterentwickeln, verhalten sollen. Was bedeutet verantwortliches Handeln in der Natur, die wir ja auch selbst sind?

\section{Literatur}

Jonas, H. (1979): Das Prinzip Verantwortung - Versuch einer Ethik für die technologische Zivilisation. Frankfurt: Suhrkamp

Jonas, H. (1993): Warum Ethik ein Thema für Technik ist: Fünf Gründe. In: Lenk, H. und Ropohl, G., Hrsg., Technik und Ethik. 2. erw. Auflage. Stuttgart: Reclam, 81-91. (engl. Original „Technology as a Subject for Ethics". Social Research, 49 (1982), 891-898).

Kant, I. (1785/1975): Grundlegung der Metaphysik der Sitten. In: Weischedel, W., Hrsg., Werke in zehn Bänden. Band 6. Darmstadt: Wissenschaftliche Buchgesellschaft.

Liebert, W. (2019, in Vorbereitung): Umwelt-Ethik. Einführung für Nicht-Philosophen.

Liebert, W. and Schmidt, J. C. (2010): Towards a prospective technology assessment: challenges and requirements for technology assessment in the age of technoscience. Poesis \& Praxis, 7, 99-116. https://doi.org/10.1007/s10202-010-0079-1.

Liebert, W. und Schmidt, J. C. (2018): Ambivalenzen im Kern der wissenschaftlich-technischen Dynamik. Ergänzende Anforderungen an eine Theorie der Technikfolgenabschätzung. TATuP, 27, 1, 52-59. https://doi.org/10.14512/tatup.27.1.52.

Ott, K., Dierks, J. und Voget-Kleschin, L. (Hrsg.) (2016): Handbuch Umweltethik. Stuttgart: Metzler.

Tugendhat, E. (1993): Vorlesungen über Ethik. Frankfurt: Suhrkamp.

UBA (Umweltbundesamt) (Hrsg.) (2004): Späte Lehren aus frühen Warnungen: Das Vorsorgeprinzip 1896-2010. Berlin. Verfügbar in: https://www.umweltbundesamt.de/publikationen/spaetelehren-aus-fruehen-warnungen [Abfrage am 11.5.2019]. 


\section{Umwelt in Gesellschaft, Politik \& Recht}

\subsection{Umwelt- und Ressourcenpolitik}

Patrick Scherhaufer, Karl Hogl, Reinhard Steurer und Helga Pülzl

Institut für Wald-, Umwelt-, und Ressourcenpolitik,

Department für Wirtschafts- und Sozialwissenschaften (WiSo)

patrick.scherhaufer@boku.ac.at, karl.hogl@boku.ac.at, reinhard.steurer@boku.ac.at, helga.puelzl@boku.ac.at

\subsubsection{Politik und Umweltpolitik - was ist das?}

Dieser Beitrag erklärt, was Politik ist und welche Dimensionen dabei zu unterscheiden sind. Darauf aufbauend werden Probleme und mögliche Lösungsansätze der Klimapolitik erläutert. Abschließend wird anhand der Elektromobilität verdeutlicht, wie damit verbundene politische Entscheidungen sowohl einer Abwägung der Umwelt- und Sozialverträglichkeit im In- und Ausland als auch einer gesellschaftlichen Akzeptanz bedürfen.

Der britische Politikwissenschafter John Kingdom (2003, S. 1) fasst das Wesen von Politik in folgender Form zusammen: „Politics is about the way people organize their lives together in a community." Diese Definition geht davon aus, dass (1) wir unser Leben nicht nur als Individuen verbringen, sondern in Gemeinschaften leben; (2) sich die Vorstellungen, wie das Zusammenleben in einer Gesellschaft organisiert werden kann und wie es zu gemeinsamen Entscheidungen kommen soll, oft deutlich voneinander unterscheiden.

Es entstehen Konflikte, weil Menschen v.a. an ihrem eigenen Nutzen oder am Nutzen derer, die ihnen nahestehen, interessiert sind oder weil sie unterschiedliche Ansichten zu den grundlegenden moralischen Fragen haben. Aufgrund knapper Ressourcen, unbegrenzter Vielfalt unterschiedlicher Meinungen, verschiedenster Interessen und ethischer Einstellungen der Menschen sind Konflikte in modernen Gesellschaften unvermeidbar. Auseinandersetzungen und Abstimmungsprozesse zwischen konkurrierenden Gruppen gehören zum Alltag demokratisch verfasster Gesellschaften (Beck und Schwarz 1995). Der Soziologe Ralf Dahrendorf (1965, zitiert in Beck und Schwarz 1995, S. 14) schreibt dazu: „Wo immer es menschliches Leben in der Gesellschaft gibt, gibt es auch Konflikt. Gesellschaften unterscheiden sich nicht darin, daß es in einigen Konflikte gibt und in anderen nicht; Gesellschaften und soziale Einheiten unterscheiden sich in der Gewaltsamkeit und der Intensität von Konflikten." Maßstab für die Güte einer politischen Ordnung ist also nicht die Häufigkeit von Konflikten in einer Gesellschaft, sondern ihre Fähigkeit, mit Konflikten umzugehen.

Dieses „Umgehen“ mit Konflikten ist nicht mit deren Lösung gleichzusetzen. Um Konflikte regeln zu können, müssen sie aber jedenfalls als solche wahrgenommen und 
konkurrierende Zielsetzungen unterschiedlicher Gruppen müssen akzeptiert werden (Dahrendorf 1992). Der deutsche Politikwissenschafter Gerhard Lehmbruch (1968, S. 17) definiert in diesem Sinne Politik als die "verbindliche Regelung gesellschaftlicher Konflikte über Werte, einschließlich materieller Güter". Die zentrale Aufgabe der Politik ist es, Angelegenheiten des Gemeinwesens mittels verbindlicher Entscheidungen im Rahmen demokratischer und rechtsstaatlicher Strukturen und Prozesse zu regeln.

Damit sind drei wesentliche Dimensionen des Politischen angesprochen: Strukturen, Prozesse und Inhalte. In der deutschen Sprache wird dies alles unter dem Begriff Politik zusammengefasst. Das Englische unterscheidet zwischen Polity, Politics und Policy:

- Politysteht für die Ordnung und die Strukturen politischer Systeme (z.B. die verfassungsrechtlich festgelegte Verteilung der Aufgaben zwischen dem österreichischen Nationalrat und den Landtagen der Bundesländer in der Gesetzgebung zu klimarelevanten Themen) und die Ordnung politischer Verfahren (Rechte und Pflichten von den an Entscheidungsprozessen beteiligten Gruppen). Interessengruppen, wie die Wirtschaftskammer und der Gewerkschaftsbund, haben z.B. bei der Regelung von Emissionen im motorisierten Individualverkehr bestimmte Anhörungs- und Mitbestimmungsrechte.

- Politics umfasst Prozesse der Konfliktaustragung und Entscheidungsfindung (Kompromiss- und Konsensbildung) zwischen konkurrierenden Gruppen. Das Spektrum reicht von politischen Auseinandersetzungen über die Definition eines Problems (z.B. welche Bedeutung hat der Klimawandel in bestimmten Weltregionen) bis zur Frage, mit welchen Ansätzen dem Klimawandel bestmöglich begegnet werden kann.

- Policy meint die inhaltlichen Aspekte von Politik. Es geht um inhaltliche Fragen der Problembearbeitung und Aufgabenerfüllung in politischen Systemen, etwa um die definierten Ziele (z.B. das Ausmaß der Reduktion von Treibhausgasemissionen) und die Wahl der Politikinstrumente, mit denen diese Aufgaben erreicht werden sollen (z.B. Verbote klimaschädlicher Produkte oder finanzielle Anreize zum Kauf emissionsarmer Fahrzeuge).

Tabelle 3.2.1 zeigt das vielfältige Spektrum umweltpolitischer Interventionsmöglichkeiten von persuasiven Politikinstrumenten über kooperative, prozedurale und marktwirtschaftliche bis hin zu regulativen Instrumenten. Instrumente in der ganz linken Spalte sollen die Bereitstellung von Informationen steuern (z.B. Gütesiegel und Umweltberichte). Ihr Einsatz bedarf nur geringer staatlicher Eingriffe. Regulative Instrumente (z.B. Gebote und Verbote) hingegen können auch mit staatlichem Zwang durchgesetzt werden. Der Staat muss die Einhaltung überwachen und Verstöße sanktionieren.

Alle drei Dimensionen der Politik sind wesentlich in der Forschung der Umweltpolitikwissenschaft, die u.a. Folgendes untersucht: Ziele der nationalen, europäischen 


\section{Umwelt in Gesellschaft, Politik \& Recht}

Tabelle 3.2.1: Das Spektrum umweltpolitischer Instrumente (Böcher und Töller 2012, S. 75)

\begin{tabular}{|c|c|c|c|c|}
\hline Persuasiv & Kooperativ & Prozedural & Marktwirtschaftlich & Regulativ \\
\hline $\begin{array}{l}\text { - Unwelt- } \\
\text { information } \\
\text { - Umweltbildung } \\
\text { - Symbole } \\
\text { (Blauer Engel) } \\
\text { - Etc. }\end{array}$ & $\begin{array}{l}\text { - Freiwillige Selbst- } \\
\text { verpflichtungen } \\
\text { - Runde Tische } \\
\text { - Dialogforen } \\
\text { - Mediation } \\
\text { - Etc. }\end{array}$ & $\begin{array}{l}\text { - Umweltverträg- } \\
\text { lichkeitsprüfung } \\
\text { - Öko-Audit } \\
\text { - Etc. }\end{array}$ & $\begin{array}{l}\text { - Umweltsteuern } \\
\text { l-abgaben } \\
\text { - Handelbare } \\
\text { Emissionsrechte } \\
\text { - Subventionen } \\
\text { - Finanzielle } \\
\text { Förderprogramme } \\
\text { - Etc. }\end{array}$ & $\begin{array}{l}\text { - Gebote/Nerbote } \\
\text { - Grenzwerte } \\
\text { (z.B. Emissionen) } \\
\text { - Bewilligungs- } \\
\text { verfahren } \\
\text { - Etc. }\end{array}$ \\
\hline
\end{tabular}

und internationalen Umweltpolitik und deren Eignung zur Problemlösung; Art, Ursache, Zeitpunkt des Instrumenteneinsatzes und Zielerreichung (Policy); geeignete Strukturen zur Problemlösung (Polity) (z.B. wer ist zur Beteiligung berechtigt? Nach welchen Regeln ist Umweltpolitik zu machen?) und Prozesse der Umweltpolitik (Politics) (z.B. welche Interessen setzen sich in Entscheidungsprozessen aus welchen Gründen durch?).

Seit den 2000er-Jahren ist das Konzept Governance in den Fokus der Politikwissenschaft gerückt. Governance geht weit über staatliche Politik hinaus und betont, dass gesellschaftlich relevante Regeln nicht nur von Regierungen, sondern auch von Unternehmen und Akteurinnen und Akteuren der Zivilgesellschaft formuliert und umgesetzt werden. Es geht dabei also um "the ways in which governing is carried out, without making any assumption as to which institutions or agents do the steering" (Gamble 2000, S. 110).

\subsubsection{Klimapolitik: Welche Probleme und mögliche Lösungen gibt es?}

Naturwissenschaftlerinnen und Naturwissenschaftler sind sich sicher: Die Klimaerwärmung schreitet in einem historisch unbekannten Tempo voran und wird überwiegend vom Menschen (genauer gesagt: durch den Ausstoß von Treibhausgasen, allen voran $\mathrm{CO}_{2}$ ) verursacht (vgl. Beitrag 4.2). Sozialwissenschaftlerinnen und Sozialwissenschaftler betonen, dass die Eindämmung dieser bedrohlichen Entwicklung zu den größten (umwelt-)politischen Herausforderungen der Menschheit gehört. Wenn aber die Bedrohung so groß und eindeutig ist, warum ist das Problem nicht längst gelöst? Dafür gibt es viele politisch relevante Gründe (Oreskes und Conway 2011):

- Der Klimawandel ist schwer wahrnehmbar und immer mehr Akteurinnen und Akteure (einschließlich hochrangige Politikerinnen und Politiker) zweifeln entweder das Phänomen insgesamt oder dessen menschliche Ursachen an. Allein da- 
raus entstehen heftige politische Auseinandersetzungen zur Sinnhaftigkeit und Angemessenheit von Klimaschutz bzw. Klimapolitik.

- Die Atmosphäre und das darin entstehende Klima ist ein globales Gemeingut (eine sogenannte Allmende), das durch Individuen oder einzelne Staaten nicht ausreichend geschützt werden kann. Klimaschutz ist nur durch eine drastische Reduktion von Treibhausgasen (v.a. $\mathrm{CO}_{2}$ ) weltweit möglich (Ostrom et al. 1999).

- Für diese sogenannte Dekarbonisierung (die Abkehr von der Nutzung kohlenstoffhaltiger Energieträger) gibt es noch keine technisch einfache und günstige Lösung (anders als z.B. bei der Bekämpfung des Ozonlochs). Die einzige praktikable Lösung ist derzeit, fossile Energiequellen (v.a. Kohle, Erdöl und Erdgas) durch erneuerbare Energie (bevorzugt Wasserkraft, biogene Energieträger, Windkraft und Photovoltaik) zu ersetzen.

- Diese sogenannte Energiewende ist wirtschaftlich und politisch schwierig, weil fossile Energie nach wie vor zu den am einfachsten verfügbaren Energiequellen zählt. Zudem sind sämtliche Systeme seit Jahrzehnten auf deren Nutzung ausgerichtet (v.a. Verkehr und die dazugehörige Tankinfrastruktur).

- Von diesen „Sachzwängen“ abgesehen, sind mit der Nutzung fossiler Energie massive unternehmerische und staatliche Interessen verbunden. So gehören z.B. Ölunternehmen wie BP zu den größten Unternehmen der Welt, und mächtige Staaten wie die USA zählen zu den weltweit größten Produktionsländern fossiler Energie.

- Viele potenzielle Verliererinnen und Verlierer einer Energiewende schüren Zweifel an der Klimaerwärmung und haben mit rechtspopulistischen Parteien wie der AfD wichtige Verbündete gefunden (Schaller und Carius 2019). Damit schließt sich der Kreis zum oben genannten ersten Grund.

Die angeführten Punkte verstärken sich gegenseitig. Dahinter stecken zahlreiche Probleme, die sich in allen Dimensionen der Politik bemerkbar machen (siehe Tabelle 3.2.2):

- Polity: Der Schutz des globalen Gemeinguts Atmosphäre bzw. Klima erfordert eine wirksame globale Regulierung. Internationale Abkommen wie jenes von Paris aus dem Jahr 2015 bestätigen zwar die Dringlichkeit dieses Anliegens, liefern aber aufgrund von gravierenden Interessenskonflikten zwischen den rund 200 Staaten völlig unzureichende Ergebnisse. Statt der in Paris geforderten Erwärmung von maximal $1,5-2{ }^{\circ} \mathrm{C}$, steuern wir derzeit auf eine Erwärmung von zumindest $3{ }^{\circ} \mathrm{C}$ zu (Falkner 2016; Spash 2016). Ambitionierte Klimaschutzinitiativen von Individuen oder einzelnen Staaten sind begrüßenswert, können das Problem aber nicht lösen, solange andere Individuen bzw. Staaten ihre Emissionen nicht ebenfalls reduzieren. Eine mögliche Lösung wäre, dass einige Länder oder Staatengemeinschaften (wie die EU) eine Vorreiterrolle im Klimaschutz 


\section{Umwelt in Gesellschaft, Politik \& Recht}

übernehmen und andere zur Nachahmung animieren bzw. drängen, z.B. durch Klimaschutzimportzölle (Nordhaus 2015).

- Politics: Akteurinnen und Akteure mit wirtschaftlichen Interessen schüren Zweifel an den Ursachen der Klimaerwärmung und behindern politische Lösungen (vgl. Oreskes und Conway 2011). Ringt sich die Politik trotz dieser Widerstände zum Klimaschutz durch, wird die Umsetzung von Maßnahmen oft durch Lobbying behindert bzw. verwässert. Für eine fortschrittlichere Klimapolitik müssen Zweifel zurückgedrängt, Problembewusstsein gefördert und öffentlicher Druck aufgebaut werden.

- Policy: Dekarbonisierung wird nicht automatisch eintreten, sondern kann nur durch den Einsatz zahlreicher politischer Maßnahmen erreicht werden. Diese Kombination umfasst u.a. Informationsinstrumente (z.B. Energielabels), Verbote (z.B. von Ölheizungen) und ökonomische Anreize (Baldwin et al. 2012). Letztere zielen v.a. darauf ab, Kostenwahrheit herzustellen, d.h., die Verursacherinnen und Verursacher von Treibhausgasen sollen die Kosten für Schäden tragen, nicht die Allgemeinheit. Diese Internalisierung externer Kosten erfolgt z.B. durch den Handel von Verschmutzungsrechten oder mittels einer $\mathrm{CO}_{2}$-Steuer. Fossilenergie würde somit teurer und im Vergleich zu erneuerbaren Energiequellen unattraktiver werden (Baranzini et al. 2017). Es gäbe also wirksame politische Maßnahmen. Je wirksamer die Maßnahmen, desto unpopulärer sind sie jedoch zumeist in der Bevölkerung - und in der Politik.

\section{Tabelle 3.2.2: Probleme und Lösungsansätze der Klimapolitik}

\begin{tabular}{|c|c|c|c|}
\hline & Charakteristika & Politische Hürden & Lösungsansätze \\
\hline Polity & $\begin{array}{l}\text { - Globales Gemeingut } \\
\text { Atmosphäre } \\
\text { - Derzeit unregulierte } \\
\text { Übernutzung in Bezug } \\
\text { auf Treibhausgase }\end{array}$ & $\begin{array}{l}\text { - Staaten mit unterschied- } \\
\text { licher sozioökonomischer } \\
\text { Entwicklung zur Dekarboni- } \\
\text { sierung verpflichten } \\
\text { - Ausreichend Fossilenergie } \\
\text { verfügbar }\end{array}$ & $\begin{array}{l}\text { Internationale Abkommen } \\
\text { abschließen, in denen entwickelte } \\
\text { Staaten mit gutem Beispiel } \\
\text { vorangehen } \\
\text { - Andere Staaten zur Nachahmung } \\
\text { von Klimaschutz animieren bzw. } \\
\text { drängen }\end{array}$ \\
\hline Politics & $\begin{array}{l}\text { - Problem schlecht } \\
\text { wahrnehmbar } \\
\text { - Zweifel an den Ursachen } \\
\text { und/oder Folgen des } \\
\text { Klimawandels }\end{array}$ & $\begin{array}{l}\text { - Dominante wirtschaftliche } \\
\text { Interessen } \\
\text { - Mangelnde öffentliche } \\
\text { Unterstützung }\end{array}$ & $\begin{array}{l}\text { - Zweifel ausräumen } \\
\text { - Problembewusstsein fördern } \\
\text { - Öffentlichen Druck aufbauen }\end{array}$ \\
\hline Policy & $\begin{array}{l}\text { Klimaschutz betrifft: } \\
\text { - Alle Bereiche des } \\
\text { Staates } \\
\text { - Die gesamte } \\
\text { Gesellschaft }\end{array}$ & $\begin{array}{l}\text { Erfolgreiche Klimapolitik } \\
\text { durch Umweltministerien } \\
\text { allein nicht möglich } \\
\text { Klimaschutz benötigt die } \\
\text { Unterstützung von anderen, } \\
\text { oft weniger interessierten } \\
\text { Ministerien }\end{array}$ & $\begin{array}{l}\text { - Maßnahmen kombinieren } \\
\text { (Labels, Verbote etc.) } \\
\text { - Treibhausgasemissionen einen } \\
\text { angemessenen Preis geben und } \\
\text { Akzeptanz dafür schaffen }\end{array}$ \\
\hline
\end{tabular}


Da Staaten mit der Einführung adäquater Klimaschutzmaßnahmen offensichtlich zögern, rücken auch in der Klimapolitik nichtstaatliche Formen der Governance in den Fokus, also Klimaschutz durch Individuen (v.a. von Konsumentinnen und Konsumenten), durch Nichtregierungsorganisationen (NGOs wie z.B. Greenpeace) und Unternehmen (z.B. durch Corporate Social Responsibility; vgl. Steurer 2013 sowie Beitrag 2.2). Allerdings: Auch nichtstaatliche Governance kämpft aufgrund der oben beschriebenen Zusammenhänge damit, die Klimaerwärmung wirksam einzudämmen.

Kurzum: Rasche Lösungen für das Problem Klimaerwärmung sind nicht in Sicht. Dies gilt besonders für eine der zentralen Herausforderungen der Dekarbonisierung: den Umstieg auf eine emissionsarme Mobilität.

\subsubsection{Mobilitätspolitik: Ein zentrales Thema der Klimapolitik}

Die regelmäßig publizierten Energieflussberechnungen zeigen, dass Österreich zu ca. $70 \%$ von fossilen Energieträgern (hauptsächlich Öl und Gas) abhängig ist (BMNT 2018). Daraus ergeben sich zwei zentrale klimapolitische Herausforderungen. Erstens, die Dekarbonisierung des österreichischen Energiesystems muss bei gleichzeitiger Sicherstellung der Energiebereitstellung geschafft werden. Zweitens, Mobilitätsinfrastruktur und das Mobilitätsverhalten müssen sich ändern, da der derzeit weitaus größte Anteil des verwendeten Öls (ca. 80\%) in der Herstellung klimaschädigender Treibstoffe für den Verkehrssektor zum Einsatz kommt. Laut Klimaschutzbericht des Umweltbundesamtes (Anderl et al. 2018, S. 106) sind zudem die Treibhausgasemissionen im Verkehrssektor seit 1990 um 66,7\% gestiegen, in allen anderen Sektoren hingegen gesunken. Das heißt, eine Energiewende bedarf einer Mobilitätswende!

Grob unterteilt gibt es drei Strategien, um Dekarbonisierung im Rahmen einer Mobilitätswende umzusetzen (vgl. Behrendt et al. 2018; Huber 1994):

- Die Konsistenzstrategie zielt darauf ab, die Qualität von Material- bzw. Energieströmen durch den Einsatz alternativer Produktionsmethoden (z.B. modulare robotergestützte Montagen), Materialien (z.B. biologische statt chemische Stoffe) und/oder Technologien (z.B. erneuerbare statt fossile Energien und entsprechende Antriebsformen) umweltfreundlicher zu gestalten.

- Unter der Effizienzstrategie werden jene Maßnahmen subsumiert, die den Materialbzw. Energieeinsatz pro Ware oder Dienstleistung durch technische Innovationen quantitativ reduzieren (z.B. durch den Einsatz effizienterer Verbrennungsmotoren oder einer ressourcenschonenden Batterieherstellung im Rahmen der Elektromobilität sowie Recycling). 


\section{Umwelt in Gesellschaft, Politik \& Recht}

- Die Suffizienzstrategie fokussiert nicht auf technische Innovationen, sondern auf eine Veränderung von Lebensstilen, Verhaltensweisen und Konsummustern (d.h. beispielsweise, verstärkter Einsatz $\mathrm{CO}_{2}$-armer Mobilitätsformen - zu Fuß gehen, Rad fahren, Nutzung öffentlicher Verkehrsmittel; das „richtige Maß“ an Reisen, Auto fahren etc.).

Die meisten EU-Länder verfolgen in Bezug auf Mobilität v.a. langfristig eine Konsistenzstrategie und greifen kurzfristig auf Effizienzstrategien zurück. Bis zu einer „flächendeckenden Elektrifizierung“ im Bereich der Mobilität hat Österreich allerdings noch einen weiten Weg vor sich. Im Jahr 2018 wurden von ca. 5 Mio. Personenkraftwagen (Pkw) erst rund 21.000 (0,4\%) elektrisch betrieben. Einen ersten Schritt zur Förderung der Elektromobilität setzte die österreichische Bundesregierung im Jahr 2016 mit einem Aktionspaket, in welchem folgende Maßnahmen vorgesehen wurden: Beim Kauf eines Elektro- oder Brennstoffzellenfahrzeugs können Privatpersonen eine einmalige Förderung in Anspruch nehmen, und private sowie öffentlich zugängliche Ladeinfrastrukturen werden unterstützt. Zudem entfällt beim Ankauf solcher Fahrzeuge die Normverbrauchsabgabe (NoVA) und die motorbezogene Versicherungssteuer.

Österreich setzt damit im Wesentlichen auf Kaufanreize bei der Beschaffung, Steuererleichterungen und auch auf Umweltinformationen (z.B. Informationsinitiative Klimaaktiv über die Vorteile der Elektromobilität, vgl. BMNT 2017), nicht aber auf andere monetäre Anreize (z.B. eine kilometerabhängige Maut für alle $\mathrm{Pkw}$ ) oder $\mathrm{Ge}$ - und Verbote (z.B. Umweltzonen bzw. Zugangsbeschränkungen für fossil betriebene Pkw).

Aus politikwissenschaftlicher Sicht lassen sich die oben beschriebenen Policies daher den marktwirtschaftlichen und persuasiven Instrumenten zuordnen (siehe Tabelle 3.2.1). Regulative Politikinstrumente spielen in Österreich derzeit eine untergeordnete Rolle.

Im Vergleich dazu hat Norwegen in Europa die weitaus höchsten Anteile elektrisch betriebener Fahrzeuge im Bestand und bei Neuzulassungen. Dies wurde mit einem umfassenden und langfristigen Plan - dem National Transport Plan 2018-2029 (Norwegian Ministry of Transport and Communications 2017) - erreicht (siehe Fallbeispiel 3.2.1).

\section{Fallbeispiel 3.2.1: Mobilitätswende in Norwegen}

Die Mobilitätswende in Norwegen basiert auf einem Instrumentenmix, der von staatlichen Investitionen in nachhaltigere Mobilitätsinfrastrukturen über Maßnahmen für mehr Sicherheit im Straßenverkehr für Kinder und Jugendliche bis hin zur Förderung der Gesundheit reicht. Im Mittelpunkt stehen neben mit fixem Ablaufdatum versehenen Fördermaßnahmen (z.B. Steuerbefreiungen) und Erleichterungen für Elektrofahrzeuge (z.B. Zugang zu Busspuren) insbesondere auch regulative Instrumente (z.B. das Verbot der Neuzulassung fossil betriebener Pkw ab 2025). 
Genauso wie die Gewinnung fossiler Brennstoffe mit Problemen verbunden ist (z.B. Umweltverschmutzung, Kriege, Einsatz neuer Technologien wie Fracking etc.), stehen Staaten, die zukünftig auf Elektromobilität setzen, neuen Herausforderungen der Umwelt- und Sozialverträglichkeit gegenüber. Derzeitige Batteriesysteme sind auf Rohstoffe wie Lithium und Kobalt angewiesen. Eine wichtige globale Lithiumrohstoffquelle liegt in der Atacama-Wüste, im Dreiländereck zwischen Chile, Bolivien und Argentinien. Für die Gewinnung des Lithiums werden große Mengen an Grundwasser benötigt. Veränderungen des Grundwasserspiegels haben aber gravierende Auswirkungen auf die lokale Flora und Fauna, die landwirtschaftliche Produktion und somit auf das Erwerbseinkommen von zumeist indigenen Kleinbäuerinnen und -bauern (Andreucci und Radhuber 2017). Kobalt kommt hauptsächlich aus der Republik Kongo. Organisationen wie Amnesty International (2017) weisen regelmäßig auf Menschenrechtsverletzungen in den großen und auch den vielen kleineren Bergbauminen hin (Kinderarbeit, unfaire bis hin zu lebensgefährliche Arbeitsbedingungen etc.). Das heißt, im Sinne einer gelungenen Konsistenzstrategie müsste aber der Einsatz der neuen Technologie „Elektromobilität“ mit der Verbesserung der sozialen und ökologischen Bedingungen in der Bergbauindustrie einhergehen.

Aufgrund der enormen Marktkonzentration (Rohstoffvorkommen in wenigen Ländern und deren Kontrolle durch einzelne Unternehmen) stellt sich die Frage, ob die Abhängigkeit von fossilen Rohstoffen nicht einfach durch die Abhängigkeit von mineralischen Rohstoffen ersetzt wird. Mögliche Auswege liegen in konsequent umgesetzten Effizienzstrategien, wobei bei der Verbreitung der Elektromobilität Folgendes sicherzustellen ist:

- Materialeinsatz von vorneherein gering halten,

- Wirkungsgrad von Batterien erhöhen,

- Batterien weiterverwenden (z.B. als stationäre Energiespeicher) und

- effizientes Recycling am Ende ihrer Lebenszeit vorantreiben.

Effizienzstrategien können aber nur einen Teil des Problems lösen, da sogenannte "Rebound-Effekte" die positiven Umweltwirkungen sehr schnell durch quantitative Gegeneffekte mindern, egalisieren oder sogar umkehren können (z.B. durch eine Steigerung der Pkw-Zulassungen). Darüber hinaus sind Elektrofahrzeuge nicht die versprochenen Null-Emissionsfahrzeuge. Bei deren Herstellung und Betrieb fallen sogenannte elsewhere emissions an: Einerseits entstehen Emissionen durch den Einsatz von nichterneuerbarem Strom; andererseits ist die Produktion von Batterien besonders energie- und damit bislang treibhausgasintensiv und mit größeren Umweltauswirkungen verbunden als die Herstellung von fossil betriebenen Fahrzeugen. Vergleicht man alternative und herkömmliche Antriebstechniken, muss die gesamte Erzeugungs- 


\section{Umwelt in Gesellschaft, Politik \& Recht}

und Verbrauchskette berücksichtigt werden. Wissenschaftlich fundierte Öko- bzw. Umweltbilanzierungen helfen bei dieser komplexen Aufgabe. Diese Untersuchungen zeigen, dass Elektromobilität (nur) mit Strom, der zu 100\% aus erneuerbaren Energiequellen stammt, eine eindeutig positive Bilanz aufweist (Wietschel et al. 2019).

Abgesehen vom in der österreichischen Klima- und Energiestrategie festgehaltenen Ziel - Bedeckung des gesamten Stromverbrauchs bis 2030 zu 100\% aus erneuerbaren Quellen - muss die Stromgewinnung aus erneuerbaren Energieträgern zusätzlich ausgebaut werden, um Elektromobilität voranzutreiben (siehe Fallbeispiel 3.2.2).

\section{Fallbeispiel 3.2.2: Rechenbeispiel Elektromobilität und Energiebedarf in Österreich}

In Österreich liegt der Anteil der Erneuerbaren bei der Stromproduktion derzeit bei knapp über 70\%.

Rechnung: Pro Elektrofahrzeug wird mit einem Alltagsstromverbrauch von $15 \mathrm{kWh} / 100 \mathrm{~km}$ und einer durchschnittlichen Fahrleistung pro Jahr von 13.000 km gerechnet. Das macht bei 5 Mio. zugelassen Pkw einen zusätzlichen Energieverbrauch von rund 10 TWh (ca. 15\% der Stromproduktion).

Fazit: Im Jahr 2017 wurden ca. 9 TWh aus Ökostromanlagen ohne Wasserkraft in das österreichische Stromnetz eingespeist. Da bei Wasserkraft nur mehr wenig Ausbaupotenzial besteht, werden für eine flächendeckende Elektrifizierung des Pkw-Bestands doppelt so viele sonstige Ökostromanlagen (Windkraft, Photovoltaik, Biomasse, Geothermie, Deponie- und Klärgas) notwendig werden. Im Bereich Windenergie würde das statt 1.200 insgesamt 2.400 Windkraftanlagen bedeuten (Stand 2017).

Neue Energieinfrastrukturprojekte und die dadurch entstehenden Energielandschaften sind jedoch mit Konflikten und Akzeptanzproblemen verbunden (Scherhaufer et al. 2017). Der Ausbau kann z.B. zu Biodiversitätsverlusten führen, die Flächenkonkurrenz zur Lebensmittelherstellung sowie zum Wohn- und Siedlungsraum erhöhen, humanökologische Einflüsse wie Lärm verstärken oder Naherholungsräume reduzieren.

Vor diesem Hintergrund wird deutlich, dass auch die Suffizienzstrategie einen wichtigen Beitrag zur Dekarbonisierung des Verkehrssektors leisten muss. Im Gegensatz zu den bisher aufgezeigten Konsistenz- und Effizienzstrategien zielt sie auf eine absolute Senkung des Ressourcen- und Energieverbrauchs ab und kritisiert das vorherrschende Paradigma eines stetigen Wachstums. Dafür müssen allerdings bisherige Selbstverständlichkeiten, Routinen und Praktiken der individuellen Mobilität hinterfragt werden. Verhaltensänderung ist jedoch ein langwieriger Prozess, der momentan in der Gesellschaft nicht nur unpopulär ist, sondern weitgehend im Zusammenhang mit dem Thema Verzicht diskutiert wird.

Aus heutiger Sicht kann nur ein Zusammenspiel von Konsistenz-, Effizienz- und Suffizienzstrategien zu einer erfolgreichen Mobilitätswende führen. Wie schon im Abschnitt 3.2.2 angedeutet, kann allein die Internalisierung externer Umweltkosten 
(z.B. mittels $\mathrm{CO}_{2}$-Steuer) die Emissionen im Verkehr reduzieren, weil Menschen auf energieintensive Mobilität verzichten (Suffizienz), Autos sparsamer werden (Effizienz) und Elektromobilität basierend auf 100\% Strom aus erneuerbaren Energiequellen verstärkt zum Einsatz kommen würde (Konsistenz).

Darüber hinaus gilt es sich stets vor Augen zu führen, dass das bisherige fossile Mobilitätssystem als einer der Hauptverursacher des Klimawandels weitaus mehr negative ökologische, soziale und wirtschaftliche Effekte generiert als eine neue Mobilitätspolitik, die Konsistenz-, Effizienz- und Suffizienzstrategien zu kombinieren versucht.

Dieser Beitrag gab Einblicke in die Herausforderungen und möglichen Problemlösungen der Umwelt- und Ressourcenpolitik im Zeitalter des Klimawandels. Er zeigte v.a. anhand der Politikfelder Klima und Verkehr auf, dass es umweltfreundliche Alternativen zum Fossilzeitalter gibt und dass diese auch politisch gefördert werden können - was bislang allerdings in Österreich nur sehr zaghaft geschieht. Gleichzeitig darf nicht übersehen werden, dass durch das Streben nach Dekarbonisierung neue Umweltprobleme und Ressourcenkonflikte entstehen werden. Dekarbonisierung möglichst umwelt- und sozialverträglich zu gestalten, erfordert systemisches Denken in komplexen Zusammenhängen, so wie es im Rahmen dieses Buches (und in interdisziplinären Umweltstudien) vermittelt werden soll.

\section{Literatur}

Amnesty International (2017): Time to recharge. Corporate action and inaction to tackle abuses in the cobalt supply chain. London. Available at: https://www.amnesty.org/en/documents/afr62/7395/2017/en/ [accessed 10.5.2019].

Anderl, M., Burgstaller, J., Gugele, B., Gössl, M., Haider, S., Heller, C., Ibesich, N., Kampel, E., Köther, T., Kuschel, V., Lampert, C., Neier, H., Pazdernik, K., Poupa, S., Purzner, M., Rigler, E., Schieder, W., Schmidt, G., Schneider, J., Schodl, B., Svehla-Stix, S., Storch, A., Stranner, G., Vogel, J., Wiesenberger, H. und Zechmeister, A. (2018): Klimaschutzbericht 2018. Report 0660. Umweltbundesamt: Wien. Verfügbar in: http://www.umweltbundesamt.at/aktuell/publikationen/publikationssuche/publikationsd etail/?pub id=2258 [Abfrage am 10.5.2019].

Andreucci, D. and Radhuber, I. M. (2017): Limits to "counter-neoliberal" reform: Mining expansion and the marginalisation of post-extractivist forces in Evo Morales's Bolivia. Geoforum, 84, 280-291. https://doi.org/10.1016/i.geoforum.2015.09.002.

Baldwin, R., Cave, M., and Lodge, M. (2012): Understanding Regulation: Theory, Strategy, and Practice. 2nd edition. Oxford: Oxford University Press.

Baranzini, A., van den Bergh, J. C., Carattini, S., Howarth, R. B., Padilla, E., and Roca, J.( 2017): Carbon pricing in climate policy: seven reasons, complementary instruments, and political economy considerations. Climate Change, 8, 3-11. https://doi.org/10.1002/wcc.462.

Beck, R. und Schwarz, G. (1995): Konfliktmanagement. Alling: Sandmann.

Behrendt, S., Göll, E. und Korte, F. (2018): Effizienz, Konsistenz, Suffizienz. Strategieanalytische Betrachtung für eine Green Economy. IZT-Text 1-2018, Berlin: IZT - Institut für Zukunftsstudien und Technologiebewertung gemeinnützige $\mathrm{GmbH}$. Verfügbar in:

https://www.izt.de/publikationen/reihe-izt-text/ [Abfrage am 13.05.2019]. 


\section{Umwelt in Gesellschaft, Politik \& Recht}

BMNT (Bundesministerium für Nachhaltigkeit und Tourismus) (2017): Vorteile der Elektromobilität. Verfügbar in: https://www.klimaaktiv.at/mobilitaet/elektromobilitaet/elektromobilitaet.html [Abfrage am 10.05.2019].

BMNT (Bundesministerium für Nachhaltigkeit und Tourismus) (2018): Energie in Österreich 2018. Zahlen, Daten, Fakten. Wien. Verfügbar in:

https://www.bmnt.gv.at/service/publikationen/energie/energie-in-oesterreich-2018.html [Abfrage am 10.5.2019].

Böcher, M. und Töller, E. (2012): Umweltpolitik in Deutschland. Eine politikfeldanalytische Einleitung. Wiesbaden: Springer VS.

Dahrendorf, R. (1992): Der moderne soziale Konflikt: Essay zur Politik der Freiheit. Stuttgart: Deutsche Verlags-Anstalt.

Falkner, R. (2016): The Paris Agreement and the new logic of international climate politics. International Affairs, 92, 1107-1125. https://doi.org/10.1111/1468-2346.12708.

Gamble, A. (2000): Economic governance. In: Pierre, J., Hrsg., Debating Governance: Authority, Steering and Democracy. Oxford: Oxford University Press, 110-137.

Huber, J. (1994): Nachhaltige Entwicklung durch Suffizienz, Effizienz und Konsistenz. In: Fritz, P., Huber, J. und Levi, H. W., Hrsg., Nachhaltigkeit in naturwissenschaftlicher und sozialwissenschaftlicher Perspektive. Stuttgart: Universitas, 31-46.

Kingdom, J. (2003): Government and Politics in Britain: An Introduction. 3rd edition. Oxford et al.: Polity Press.

Lehmbruch, G. (1968): Einführung in die Politikwissenschaft. Stuttgart, Berlin, Köln, Mainz: Kohlhammer.

Nordhaus, W. D. (2015): Climate clubs: Overcoming free-riding in international climate policy. American Economic Review, 105, 1339-1370. https://doi.org/10.1257/aer.15000001.

Norwegian Ministry of Transport and Communications (2017): Meld. St. 33 (2016-2017) Report to the Storting (white paper). National Transport Plan 2018-2029. A targeted and historic commitment to the Norwegian transport sector (English summary). Available at: https://www.regjeringen.no/en/dokumenter/meld.-st.-33-20162017/id2546287/ [accessed 10.5.2019].

Oreskes, N. and Conway, E. M. (2011): Merchants of Doubt: How a Handful of Scientists Obscured the Truth on Issues from Tobacco Smoke to Global Warming. New York: Bloomsbury Press.

Ostrom, E., Burger, J., Field, C. B., Norgaard, R. B., and Policansky, D. (1999): Revisiting the commons: Local lessons, global challenges. Science, 284, 278-282.

https://doi.org/10.1126/science.284.5412.278.

Schaller, S. and Carius, A. (2019): Convenient truths: Mapping climate agendas of right-wing populist parties in Europe. Berlin: adelphi consult $\mathrm{GmbH}$. Available at: https://www.adelphi.de/de/publikation/convenient-truths [accessed 10.5.2019].

Scherhaufer, P., Höltinger, S., Salak, B., Schauppenlehner, T., and Schmidt, J. (2017): Patterns of acceptance and non-acceptance within energy landscapes: A case study on wind energy expansion in Austria. Energy Policy, 109, 863-870. https://doi.org/10.1016/j.enpol.2017.05.057.

Spash, C. L. (2016): This changes nothing: The Paris Agreement to ignore reality. Globalizations, 13, 928-933. https://doi.org/10.1080/14747731.2016.1161119.

Steurer, R. (2013): Disentangling governance: a synoptic view of regulation by government, business and civil society. Policy Sciences, 46, 387-410. https://www.jstor.org/stable/42637288.

Wietschel, M., Kühnbach, M. und Rüdiger, D. (2019): Die aktuelle Treibhausgasemissionsbilanz von Elektrofahrzeugen in Deutschland. Working Paper Sustainability and Innovation, No. S 02/2019. Karlsruhe: Frauenhofer ISI. Verfügbar in:

http://publica.fraunhofer.de/dokumente/N-537432.html [Abfrage am 10.5.2019]. 


\subsection{Soziologie des Umweltverhaltens}

Christine Altenbuchner und Ulrike Tunst-Kamleitner

Institut für Nachhaltige Wirtschaftsentwicklung,

Department für Wirtschafts- und Sozialwissenschaften (WiSo)

christine.altenbuchner@boku.ac.at, ulrike.tunst@boku.ac.at

\subsubsection{Subjektives Umweltbewusstsein und Umweltverhalten}

\subsubsection{Determinanten des Umweltverhaltens}

Warum denken Menschen zwar an Umweltschutz, schädigen die Umwelt aber trotzdem? Warum müssen Menschen in der US-Stadt Flint mit Blei verseuchtes Wasser trinken, und was hat das mit den Schülerinnen- und Schülerprotesten von Fridays for Future zu tun? Warum ist Greta Thunberg („Ich habe gelernt, dass man niemals zu klein ist, um einen Unterschied zu machen.") Heldin der Klimaschutzbewegung? Und was hat das alles mit Umweltsoziologie und nachhaltiger Entwicklung zu tun?

SDG 16 soll „friedliche und inklusive Gesellschaften für eine nachhaltige Entwicklung fördern“. Wie können wir das Ziel erreichen und den Wandel hin zu einer umweltbewussteren Gesellschaft schaffen? Seit den 1990er-Jahren steigt das Umweltbewusstsein stetig. Individuen halten die Umwelt für schützenswert und zeigen dementsprechende Handlungsbereitschaft. Dieses Bewusstsein wird jedoch kaum oder gar nicht in Verhalten umgesetzt. Menschen fahren weiter mit Verbrennungskraftmotoren, obwohl sie wissen, dass diese $\mathrm{CO}_{2}$ ausstoßen. Sie kaufen nicht (nur) regionale BioLebensmittel, obwohl sie die Umwelt schützen möchten. Sie lassen Geräte im Stand-byModus laufen, obwohl damit Energie nutzlos verbraucht wird. Die Umweltsoziologie untersucht diese Schere zwischen Einstellung und Handeln systematisch und unterscheidet dafür verschiedene Komponenten.

Der Begriff Umwelteinstellung wird synonym zum Begriff Umweltbewusstsein verwendet. Es setzt sich aus drei Komponenten zusammen:

- Das Umweltwissen einer Person, das ist die kognitive Komponente: „Was weiß ich bzw. was will ich über die Umweltauswirkungen meines Verhaltens wissen?“.

- Die subjektive Betroffenheit einer Person von Umweltproblemen, das ist die emotionale oder affektive Komponente: „Bin ich direkt oder indirekt von Umweltauswirkungen betroffen?“”.

- Die Verhaltensbereitschaft einer Person der Umwelt oder Umweltproblemen gegenüber, das ist die Handlungs- oder Reaktionskonsistenz. Man spricht auch von der handlungsorientierten oder konativen Komponente: „Will ich mich umweltbewusst verhalten?"“. 


\section{Umwelt in Gesellschaft, Politik \& Recht}

Die drei Komponenten umfassen das Denken, Fühlen und Reagieren einer Person. Einstellungen können demzufolge als Mindset von einzelnen Überzeugungen verstanden werden, die zusammen die Einstellung als insgesamt positive oder negative Bewertung bilden.

Bei Einstellungen ist zwischen innerer oder interner bzw. äußerer oder externer (z.B. monetärer Nutzen und Kosten) Verhaltenskontrolle bzw. zwischen intrinsischer und extrinsischer Motivation zum Handeln zu unterscheiden.

Abbildung 3.3.1 zeigt Einflussgrößen des subjektiven Umweltverhaltens, das zusätzlich zu den Einstellungskomponenten die soziale Einbettung des Verhaltens einbezieht. Eine zentrale Rolle spielt hier die Verhaltensintention (konative Einstellungskomponente), das direkte Bestimmungselement des Verhaltens, die Verhaltensmotivation bzw. -demotivation. Die Verhaltensintention wiederum wird von folgenden Faktoren bestimmt:

- von den kognitiven (Umweltwissen) und emotionalen (Betroffenheit) Einstellungskomponenten,

- von der sozialen Einbettung des Verhaltens, also vom Einfluss, den die Meinung von uns wichtigen Personen über unser erwünschtes Verhalten auf unsere Intention hat,

- von der (subjektiv) wahrgenommenen Verhaltenskontrolle. Hier geht es darum, wie leicht oder schwierig es scheint, ein Verhalten durchzuführen; darüber entscheidet unsere Wahrnehmung verfügbarer bzw. begrenzender Ressourcen in Form von Zeit, Geld und Zusammenarbeit mit anderen (Vogel 1999).

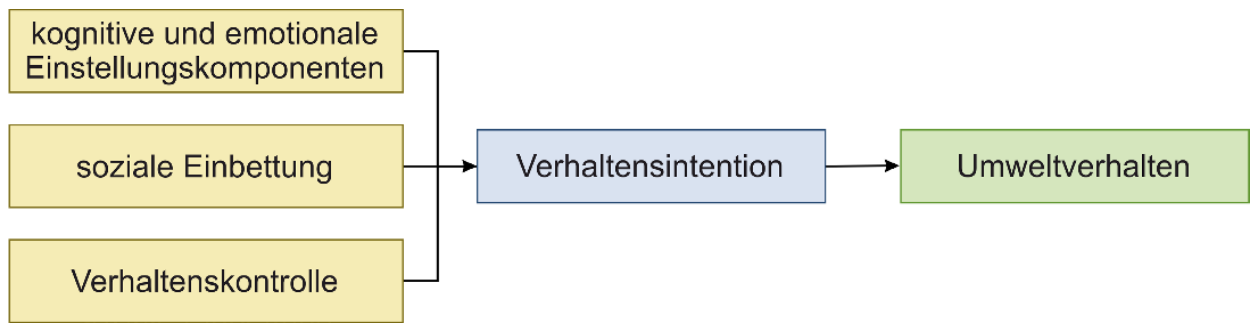
Abbildung 3.3.1: Einfluss von Umwelteinstellungen im Kontext anderer
Bestimmungsfaktoren des Umweltverhaltens

Wahrnehmung setzt sich aus wahr und nehmen zusammen - wir nehmen etwas als wahr an, die Wahrheit kann aber durchaus anders liegen. Dies gilt auch für die kognitive Einstellungskomponente. Wir nehmen die Welt nicht direkt, sondern immer vermittelt wahr. Die Vermittlung der Realität erfolgt über unsere Sinne und über sozial begründete Wahrnehmungsmuster. Wahrnehmung ist immer eine Selektion von Information. In Zeiten von Fake News und Breitbart News Network lässt sich das leicht erklären: Jede/Jeder kreiert ihre/seine eigene Wahrheit, die ihr/ihm am besten entgegenkommt und am ehesten ihren/seinen vorhandenen Wahrnehmungsmustern entspricht. Was wir aus der Realität als Information aufnehmen, wird davon beein- 
flusst, welche Auswahlmuster wir durch Erziehung und durch die Einflüsse anderer Menschen und Institutionen zugrunde legen.

\subsubsection{Zwischen Bewusstsein und Verhalten}

Warum Bewusstsein und Verhalten oft diametral zueinander stehen, dazu gibt es keine einheitliche Theorie, die für alle Bereiche und Verhaltensweisen eine plausible Erklärung liefern würde. Infolge werden einige Erklärungsansätze vorgestellt (Diekmann und Preisendörfer 2001; Kuckartz 2008):

- Bei einzelnen Problemen gibt es nicht genügend Information darüber, wie man sich umweltverantwortlich verhalten kann.

- Für die Bevölkerung sind die Hauptverursacher von Umweltproblemen meist Institutionen (nicht Individuen). Individuen glauben, dass Institutionen beim Umweltschutz die Führung übernehmen sollen: „Ich kann sowieso nichts machen. Warum soll ich aufs Autofahren verzichten, wenn die Industrie vielmehr $\mathrm{CO}_{2}$ produziert als ich. Soll die Politik das doch verbieten!“

- Ein weiteres grundlegendes Problem sind ökonomische Aspekte (Verhaltenskontrolle). Menschen mögen bereit sein, einige Aspekte ihres Lebens zu verändern, andere jedoch nicht. Wenn umweltorientiertes Verhalten wenig kostet, wie etwa Mülltrennen, führt Umweltbewusstsein leichter zu entsprechendem Verhalten. Den Bus statt das Auto zu nehmen, kostet viel mehr Zeit und mag unbequemer sein, hier gibt es weniger Veränderungsbereitschaft.

- Für jedes Individuum scheint eine Verbesserung der Umwelt v.a. das Handeln der anderen zu erfordern. Dem oder der Einzelnen scheint nur ein geringer Beitrag möglich, ja, sie können sogar die Chance sehen, sich um die Kosten zu drücken (Trittbrettfahrer). „Sollen doch meine Nachbarn mit dem Bus fahren, die haben nicht so weit in die Arbeit. Für mich ist das viel aufwendiger."

- Die Konformitätsbereitschaft des Individuums in Hinblick auf das soziale Bezugssystem und die soziale Einbettung kann gering sein, der normative Einfluss auf das Individuum ist dann nur schwach. Individuen können entscheiden, sich gar nicht so verhalten zu wollen, wie andere es gerne hätten. Die Meinung anderer kann Einzelnen egal sein, sie wollen ihr eigenes Ding machen.

- Das Verhaltensangebot kann fehlen. Wo überhaupt kein Bus fährt, stellt sich die Frage gar nicht.

Umweltorientierte Verhaltensänderungen können zu Zielkonflikten führen. Möglichst schnell und günstig nach Berlin zu kommen, gleichzeitig aber die Umwelt zu schonen, wirft die Frage „Zug oder Flug“ auf. Umweltbewusst zu handeln kann zusätzliche Kosten verursachen, etwa durch Teilnahme an freiwilligen Aktionen, aber 


\section{Umwelt in Gesellschaft, Politik \& Recht}

auch zusätzlichen Nutzen bringen. Durch die Teilnahme an Klimaprotesten ist es möglich, viele Freunde außerhalb der Schule zu treffen.

Externe Faktoren (etwa finanzielle Komponenten) können die Wirkung von Einstellungen begrenzen. Es stellt sich die Frage: Wie groß muss das Umweltbewusstsein sein, um die Hürde bestimmter Verhaltenskosten (z.B. Zeit oder finanzielle Verluste) zu nehmen? Setzt man externe Anreize, ist nach den Erkenntnissen der Sozialpsychologie ein Nachzieheffekt des Bewusstseins anzunehmen. Wenn eine Person in ihrer Arbeit als Marketingassistentin bzw. -assistent grüne Produkte bewirbt, obwohl sie gar kein Öko-Fan ist, entsteht Dissonanz. Einstellungsdiskrepantes Handeln erzeugt ein unangenehmes Gefühl. Erzwingt ein verhaltenssteuernder Faktor (in diesem Fall das Gehalt) ein bestimmtes Verhalten, wird sich über kurz oder lang die Einstellung verändern. Das heißt, wenn jemand lange Zeit grüne Produkte bewirbt und dafür argumentiert, ist es wahrscheinlich, dass diese Einstellung in sein Wertesystem übernommen wird. Ändert sich das Verhalten, verändern sich auch grundlegende Werthaltungen und Einstellungen, welche zukünftiges Verhalten autonom in die gewünschte Richtung lenken können.

Ist der finanzielle Anreiz im Vergleich zu den zusätzlichen Kosten des Zielverhaltens groß und berücksichtigt man vorhandenes Umweltbewusstsein nicht, kann es zu einer „Überrechtfertigung“ kommen. Die intrinsische Motivation wird durch eine extrinsische Motivation ersetzt, ein Verdrängungseffekt tritt auf. Wenn ein bäuerlicher Betrieb Felder extensiv bewirtschaftet, erhält er eine Agrarförderung. Wenn die Betriebsleiterin oder der Betriebsleiter zunächst diese Art der Bewirtschaftung auch ohne Fördergelder durchführen würde, kann ein paradoxer Effekt auftreten. Werden die Fördergelder eingestellt, geht der Anreiz, die Felder weiter extensiv zu bewirtschaften, verloren, die intrinsische Motivation ist verschwunden.

Umwelteinstellungen und ihr Zusammenhang mit Umweltverhalten sind eines der Hauptforschungsgebiete der Umweltsoziologie. In der Fachsprache - im Gegensatz zur Alltagssprache - ist Umweltbewusstsein nicht mit umweltgerechtem Verhalten gleichgesetzt. Umweltgerechtes Verhalten ist ein Teil des Alltagsverhaltens. „Umweltgerecht verhält sich jemand nicht nur aus Einsicht und rationaler Entscheidung, sondern möglicherweise auch aus Knappheit an finanziellen Mitteln ... oder vielleicht aus bloßer Gewohnheit. Das sind Personen mit umweltgerechtem Verhalten ohne entsprechende Einstellungen“" (Kuckartz 2008, s.p.).

\subsubsection{Umweltgerechtigkeit}

Menschen sind von Umweltbelastungen nicht gleich stark betroffen, auch nicht innerhalb eines Landes. In den USA sind v.a. afroamerikanische und ärmere Bevölkerungsschichten von Umweltbelastungen überproportional betroffen (siehe Fallbeispiel 3.3.1). 
Flint, eine Kleinstadt in Michigan, $95 \mathrm{~km}$ nordwestlich von Detroit, prosperierte bis vor wenigen Jahrzehnten. Nachdem die Automobilindustrie ihre Produktion verlagerte, blieben hohe Arbeitslosenraten sowie Armut. Die Bevölkerungszahl der Stadt ist seither drastisch gesunken: Wer kann, zieht weg. In der Stadt wohnen mehrheitlich Afroamerikanerinnen und Afroamerikaner.

Die Stadt ist hochverschuldet, weshalb vom Gouverneur ein Notfallfinanzverwalter, Michael Brown, eingesetzt wurde - was nur bei Orten mit afroamerikanischer Bevölkerung zu geschehen scheint, wie es später als Vorwurf formuliert wurde. Im April 2014 traf Brown eine folgenschwere Entscheidung: Um Geld zu sparen, sollte Wasser nicht mehr aus Detroit, sondern aus dem regionalen Huron-See bezogen werden. Da noch keine Pipeline existierte, wurde übergangsweise Wasser aus dem Flint River gewonnen.

Bereits nach wenigen Tagen klagten die Bewohnerinnen und Bewohner über Hautausschläge, Übelkeit und Müdigkeit. Trotz Nachfragens gab es keine Reaktion der Verwaltung. Monate später kam der Verdacht auf, dass das Wasser Blei enthalten könnte. Michigans Umweltbehörden wiegelten ab. Eine Rückkehr zum Wasser aus Detroit lehnte man aus Geldmangel ab. Stattdessen wurde eine Kommission zur Verbesserung der Wasserqualität eingesetzt. Diese verkündete kurz darauf, dass die Untersuchung keine Auffälligkeiten gezeigt habe. Später stellte sich heraus, dass die Tests nicht sachgemäß durchgeführt worden waren. Ein Mitarbeiter der Umweltbundesbehörde EPA (Environmental Protection Agency) warnte in einem internen Bericht vor Problemen mit dem Trinkwasser. Dieser Bericht wurde ignoriert.

Eine in Flint praktizierende Ärztin stellte erhöhte Bleiwerte im Blut einiger Kinder fest, es kam zu Haarausfall und Ausschlägen. Mehr als eineinhalb Jahre lang tranken die Bewohnerinnen und Bewohner bleiverseuchtes Wasser, bis Wissenschaftlerinnen und Wissenschaftler flächendeckend einen zu hohen Bleigehalt feststellten und mit diesen Erkenntnissen an die Öffentlichkeit gingen. Erst das veranlasste den Gouverneur dazu, Wasser wieder aus Detroit zu beziehen. Die Langzeitfolgen für die 100.000 Bewohnerinnen und Bewohner sind unklar.

Was war geschehen? Das Wasser des Flint River griff die alten Bleirohre der Stadt an. Da man auf die Beigabe von Chemikalien, die dies verhindert hätten, verzichtete - die Maßnahme hätte 100 Dollar pro Tag gekostet - wurde Blei freigesetzt. Hätten die Behörden früher reagiert, wenn Flint "weiß" und „reich" wäre? Die Betroffenen sind mehrheitlich Afroamerikanerinnen und Afroamerikaner, Flint ist ein klassischer Fall von Environmental Injustice oder gar von Environmental Racism.

Die soziale Ungleichverteilung von Umweltbelastungen ist bereits seit den 1970erJahren ein Thema. Es hat seinen Ursprung nicht in der Wissenschaft, sondern in der US-amerikanischen Bürgerrechtsbewegung (Elvers 2007). Seit Jahrzehnten kämpfen in den USA ethnische Minoritäten und Arme gegen eine systematisch höhere Umweltbelastung. Es geht um Umweltgerechtigkeit (Environmental Justice), die soziale bzw. sozialräumliche Ungleichverteilung von Umweltbelastungen wird kritisiert. Man unterscheidet zwischen intragenerationeller Gerechtigkeit (innerhalb einer Generation, wie etwa im Fall Flint) und intergenerationeller Umweltgerechtigkeit (zwischen zwei oder mehreren Generationen) (Glotzbach und Baumgartner 2012). SDG 10 (Ungleichheit innerhalb von und zwischen Staaten verringern) und die Schülerinnen- und Schülerproteste Fridays for Future um Greta Thunberg (2019) fordern sowohl intragenerationelle Umweltgerechtigkeit („Unsere Zivilisation wird für das Wohl einer 


\section{Umwelt in Gesellschaft, Politik \& Recht}

sehr kleinen Gruppe von Menschen geopfert, damit letztere immer mehr Geld erwirtschaften können.") als auch intergenerationelle Umweltgerechtigkeit („Ihr sagt, dass ihr eure Kinder über alles liebt. Und trotzdem stehlt ihr ihnen ihre Zukunft, direkt vor ihren Augen."). Das Thema Gerechtigkeit und die Verantwortung für Umweltbelastungen ist immer auch eine ethische Frage (siehe dazu Beitrag 3.1).

\subsubsection{Gesellschaftliche Resonanz}

Bis in die 1960er- und 1970er-Jahre fand das Thema Natur und Umwelt in der Öffentlichkeit, aber auch in der Soziologie, kaum Beachtung. Erst mit der Entstehung von Umweltbewegungen und öffentlichen Debatten über ökologische Probleme begannen Soziologinnen und Soziologen sich mit Umweltthemen zu beschäftigen. Im Zentrum der Analysen stehen folgende Themen (Diekmann und Preisendörfer 2001):

- ökologische Folgen und Nebenfolgen von Handlungen individueller Akteurinnen und Akteure (Personen) und korporativer Akteure (Firmen, Verbände, Organisationen),

- Bedingungen, die dazu führen, dass Veränderungen in der Umwelt der Menschen als ökologisches Problem erkannt werden,

- gesellschaftliche Reaktionen auf ökologische Probleme (Proteste, Umweltbewegung, nationale und internationale Umweltpolitik).

Die Art der gesellschaftlichen Reaktion auf Umweltveränderungen wird im Wesentlichen beeinflusst von: (1) der Stärke und Relevanz der Umweltveränderung, (2) den gesellschaftlichen Wahrnehmungs- und Bewertungsmustern der Umweltveränderung und (3) der Verwundbarkeit und Sensibilität sozialer Systeme sowie der gesellschaftlichen Reaktions- und Anpassungsmöglichkeiten. Die soziale Relevanz eines Ereignisses hängt nicht allein von seiner Stärke ab. Tatsache ist, dass (nach Brand und Reusswig 2007)

- sich viele ökologische Probleme der alltäglichen Wahrnehmbarkeit entziehen, so auch der anthropogene Klimawandel,

- die Komplexität der verursachenden Prozesse hoch ist,

- sich Probleme mit hoher individueller Betroffenheit leicht politisieren lassen und

- Massenmedien die öffentliche Meinung stark beeinflussen.

In der Vergangenheit wurden Veränderungen in der öffentlichen Debatte und in weiterer Folge in der Politik und in der politischen Landschaft durch Umweltbewegungen erreicht. Beispielhaft für Österreich sind das Verbot von Atomkraft als Errungenschaft der Anti-Atomkraft-Bewegung und der Proteste gegen das AKW Zwentendorf 1978 sowie die Gründung der Grünen Partei als Folge der Proteste gegen das Donaukraftwerk Hainburg 1984. Auch in der Klimawandeldebatte zeigt sich ein ähnliches Bild. Die ökologischen Folgen und Nebenfolgen unseres Lebenswandels auf das Klima sind weit- 
gehend bekannt (siehe Beitrag 4.2). Die Reaktionen darauf können bei den gesellschaftlichen Akteurinnen und Akteuren unterschiedlich ausfallen. Die internationale Politik hat mit dem Kyoto-Protokoll im Jahr 1997 einen wichtigen Schritt zur Reduktion der Treibhausgase gesetzt. Infolge wurden Emissionshandelssysteme etabliert und weitere Übereinkommen (wie Paris 2015) beschlossen. Auch die nationale Politik reagiert beispielsweise mit Strategieplänen zur Anpassung an den Klimawandel. Die Grundlagen für Anpassungsstrategien liefert die Wissenschaft. Gesellschaftlicher Protest spiegelt sich in internationalen Netzwerken wie z.B. dem Climate Action Network (CAN) wider.

\section{Fallbeispiel 3.3.2: Greta Thunberg - eine Heldin?}

Neue Impulse erhielt der zivilgesellschaftliche Protest mit der von Greta Thunberg initiierten Bewegung „Fridays for Future“. Ihr Aktionismus „Klimaschutzprotest statt Schule am Freitag" hat 2018 große Aufmerksamkeit erfahren und gipfelte am 15. März 2019 im weltweiten Klimastreik von Schülerinnen und Schülern. Greta Thunberg wurde für viele junge Menschen weltweit zum Vorbild und zur Heldin und löste eine starke Mobilisierungswelle aus. Umweltbewegungen und zivilgesellschaftlicher ökologischer Protest sind ein wichtiger Schritt zur Veränderung von Verhalten und von Strukturen. Welche politischen und gesellschaftlichen Veränderungen die derzeitigen Proteste langfristig auslösen werden, wird erst die Zukunft zeigen.

\subsubsection{Arbeitsfelder der Umweltsoziologie}

Umweltsoziologie befasst sich mit dem Verhältnis von Mensch und Gesellschaft zu Natur und Umwelt. Brand (1997) unterscheidet drei Perspektiven:

- Beobachterperspektive: Die Umwelt wird als gesellschaftliches Diskurs- und Konfliktfeld gesehen. Diese klassische soziologische Perspektive richtet den beobachtenden Blick auf die Gesellschaft. Sie zeigt verschiedene Blickwinkel von Akteurinnen und Akteuren sowie Konfliktebenen. Naturalistische Deutungen (wie ökologische Grenzen) werden kulturellen Konstruktionen und Bedeutungen gegenübergestellt. Gefragt wird, wie sich Umweltbewegungen und ihre Aktionsformen analysieren lassen (siehe Abschnitt 3.3.3), ob Umweltbewusstsein ein Wohlstandsphänomen ist oder inwieweit Umweltbewusstsein das Alltagsverhalten beeinflusst (siehe Abschnitt 3.3.1).

- Problembezogene Perspektive: Betrachtet werden gesellschaftliche Ursachen von Umweltschäden und praktische Interventionsmöglichkeiten. Gefragt wird etwa, ob nachhaltige Entwicklung möglich ist und wie man in Gesellschaften entsprechend eingreifen kann.

- Reflexive Perspektive: Welchen Beitrag kann die Umweltsoziologie zur Lösung von Umweltproblemen leisten? Gefragt wird, wie Soziologie und Gesellschaftstheorien mit der Natur umgehen, oder welche erkenntnistheoretischen Grundmodelle sich dafür eignen, die Gesellschaft als Verursacher von Umweltschäden abzubilden. 


\section{Umwelt in Gesellschaft, Politik \& Recht}

Wenn die Gesellschaft in ihrer natürlichen Umwelt Störungen auslöst, die ihren Weiterbestand gefährden können, dann bedarf es sozialwissenschaftlicher Kompetenz, um

- das störende gesellschaftliche Verhalten zu beobachten und seine Ursachen zu erkennen (gesellschaftliche Selbstbeobachtung),

- geeignete Interventionsmöglichkeiten zu erkennen und

- dies verschiedenen Akteurinnen und Akteuren bewusst zu machen, durch Aufklärung, Bildung, Beratung und Entwicklung.

Mit der Analyse von Einflussfaktoren subjektiven Umweltbewusstseins und Umweltverhaltens, mit Studien zur Umweltgerechtigkeit und der gesellschaftlichen Wahrnehmung sowie der Resonanz von Umweltproblemen leistet die Umweltsoziologie ebenso wie andere Disziplinen im UBRM einen Erklärungs- und Lösungsbeitrag zu den Phänomenen und Herausforderungen unserer Zeit. Um Umweltprobleme lösen zu können bzw. sie erst gar nicht entstehen zu lassen, braucht es die Zusammenarbeit verschiedener wissenschaftlicher Disziplinen (Interdisziplinarität) und die Zusammenarbeit der Wissenschaftlerinnen und Wissenschaftler mit Akteurinnen und Akteuren in den verschiedenen gesellschaftlichen Subsystemen (Transdisziplinarität), etwa mit Kommunen, umweltpolitischen Einrichtungen, NGOs, Institutionen des Bildungssystems und der Industrie.

\section{Literatur}

Brand, K.-W. (Hrsg.) (1997): Nachhaltige Entwicklung. Eine Herausforderung an die Soziologie. Opladen: Leske\&Budrich.

Brand, K. W. und Reusswig, F. (2007): Umwelt. In: Joas, H., Hrsg., Lehrbuch der Soziologie. Frankfurt, New York: Campus Verlag. 653-672.

Diekmann, A. und Preisendörfer, A. (2001): Umweltsoziologie. Eine Einführung. Reinbek bei Hamburg: Rowohlt Taschenbuch Verlag GmbH.

Eligon, J. (2016): A question of environmental racism in Flint. New York Times. Available at: https://www.nytimes.com/2016/01/22/us/a-question-of-environmental-racism-in-flint.html [accessed 8.4.2019].

Elvers, H.-D. (2007): Umweltgerechtigkeit als Forschungsparadigma der Soziologie. Soziologie, 36, $1,21-44$.

Glotzbach, S. and Baumgartner, S. (2012): The relationship between intragenerational and intergenerational ecological justice. Environmental Values, 21, 3, 331-355. https://www.jstor.org/stable/23240649.

Katner, A., Pieper, K. J., Lambrinidou, Y., Brown, K., Hu, C. Y., Mielke, H. W., and Edwards, M. A. (2016): Weaknesses in federal drinking water regulations and public health policies that impede lead poisoning prevention and environmental justice. Environmental Justice, 9, 4, 109-117. https://doi.org/10.1089/env.2016.0012.

Kuckartz, U. (2008): Umweltbewusstsein und Umweltverhalten. Bonn: Bundeszentrale für politische Bildung bpb. Heft 287. Verfügbar in: https://www.bpb.de/izpb/8968/umweltpolitik [Abfrage am 8.4.2019]

Kuhn, J. (2016): Verseuchtes Wasser - Flint im US-Bundesstaat Michigan: 100000 Menschen vergiftet. Die Süddeutsche. Verfügbar in: https://www.sueddeutsche.de/panorama/verseuchteswasser-flint-im-us-bundesstaat-michigan-menschen-vergiftet-1.2827781 [Abfrage am 8.4.2019]

Thunberg, G. (2019): Greta Thunberg COP24 statement. Available at: https://youtu.be/e68Hie0J5E [accessed 8.4.2019]

Vogel, S. (1999): Umweltbewußtsein und Landwirtschaft. Theoretische Überlegungen und empirische Befunde. Weikersheim: Margraf Verlag. 


\title{
3.4 Umweltgeschichte und Erdzukunft
}

\author{
Martin Schmid und Verena Winiwarter \\ Institut für Soziale Ökologie, \\ Department für Wirtschafts- und Sozialwissenschaften (WiSo) \\ martin.schmid@boku.ac.at, verena.winiwarter@boku.ac.at
}

\subsubsection{Langfristige Perspektiven für eine nachhaltige Welt}

Umweltgeschichte untersucht die Vergangenheit um der Zukunft willen. Sie befasst sich einerseits mit der Rekonstruktion von Umweltbedingungen in der Vergangenheit: Wie sah eine Landschaft einmal aus? Wie wurde sie genutzt? Wo verlief früher ein Fluss? Was für ein Wald war das vor 200 Jahren? Sie muss sich dafür andererseits mit der Rekonstruktion der Wahrnehmung und Interpretation dieser vergangenen Umwelten durch die damals lebenden Menschen beschäftigen. Wie haben Menschen etwa Berge wahrgenommen, als Bedrohung oder als Freizeitareal, und wann und warum hat sich das geändert? Kurz gesagt befasst sich Umweltgeschichte mit den Wechselbeziehungen zwischen Menschen und dem Rest der Natur - weil Menschen selbst Natur sind, ihr aber zugleich gegenüberstehen, in Natur eingreifen, sie verändern müssen, um leben zu können. Umweltgeschichte ist ein interdisziplinäres Fach, das Zugänge aus den Kultur-, Sozial-, Natur- und Ingenieurwissenschaften miteinander verbindet (Winiwarter und Knoll 2007).

Die globalen Herausforderungen, wie sie zuletzt in der UN-Agenda 2030, den SDGs, formuliert worden sind, haben eine Geschichte. Was waren die Triebkräfte, die Probleme wie globale Ungleichheiten (SDG 10) oder nichtnachhaltige Produktions- und Konsummuster (SDG 12) hervorgebracht haben, um deren Lösung heute mit solchen Initiativen gerungen wird? Wann und wie ist diese - menschheitsgeschichtlich außerordentliche - Situation entstanden?

Auch die Nachhaltigkeitsdebatte selbst hat eine Geschichte, in der Aufmerksamkeit verschieden verteilt ist, in der manche Interessensgruppen eine Stimme bekommen haben und sich Gehör verschaffen konnten und andere außen vor gelassen wurden. Die Nachhaltigkeitsdebatte hat sich mit jedem Meilenstein verändert: von der ersten Konferenz der Vereinten Nationen zum Thema Umwelt 1972 in Stockholm, über den Brundtland-Bericht mit seiner bis heute nachwirkenden Nachhaltigkeitsdefinition von 1987, die „Rio-Konferenz“ 1992 und die „Millennium Development Goals“ von 2000 bis zu den heute die Debatte dominierenden SDGs. Immer noch geht es um Entwicklung, wie 1987: "Sustainable development is development that meets the needs of the present without compromising the ability of future generations to meet their 


\section{Umwelt in Gesellschaft, Politik \& Recht}

own needs" (WCED 1987, S. 43). Mit einem Abstand von bald fünf Jahrzehnten muss man konstatieren: Die meisten Maßnahmen, die zu nachhaltiger Entwicklung führen sollten, blieben halbherzige Kurskorrekturen in kleinem Maßstab. Auch deshalb ist der Begriff „Nachhaltigkeit“ in die Krise geraten, nach seiner recht steilen Karriere in den öffentlichen Debatten ab den 1970er-Jahren.

Umso bemerkenswerter war 2011 die Publikation des Jahresgutachtens des Wissenschaftlichen Beirats der Bundesregierung Globale Umweltveränderungen (WBGU) in Deutschland, das erstmals in dieser Deutlichkeit von der Notwendigkeit einer „Großen Transformation" sprach. Der WBGU sieht die Transformation zu einer nachhaltigen, klimaverträglichen Gesellschaft als offenen Suchprozess. Zwar ließen sich Nachhaltigkeitsziele benennen, eine genaue Beschreibung eines anzustrebenden Endzustands von Wirtschaft und Gesellschaft sei aber nicht möglich (WBGU 2011). Der WBGU diagnostizierte also, dass wir zwar das Ziel kennen, aber nicht den Weg oder die mit dem Ziel verbundenen, konkreten gesellschaftlichen Veränderungen.

Als historische Spezialisierung der Sozialen Ökologie (Haberl et al. 2016) kann Umweltgeschichte dazu beitragen, sich in diesen großen Fragen zu orientieren. Wer umwelthistorisch informiert ist, denkt anders über die Bedingung der Möglichkeit einer nachhaltigen Entwicklung nach. Nicht zuletzt lehrt der Blick in die Vergangenheit, dass wir uns keine zu simplen und linearen Vorstellungen machen sollten - von Gesellschaft und Natur und davon, wie das eine auf das andere wirkt. Solche grundsätzlichen Einsichten sind wichtig, um die Rolle als Expertin oder Experte verantwortungsvoll wahrnehmen und zur Lösung drängender Nachhaltigkeitsprobleme in der beruflichen Praxis beitragen zu können.

\subsubsection{Gesellschaftlicher Wandel ist Wandel des Umgangs mit Natur}

Seit Menschen vor etwa 10.000 Jahren zunächst im „fruchtbaren Halbmond“ sesshaft wurden, waren die meisten von ihnen als Viehzüchter, Ackerbauern oder Fischer tätig. Das blieb bis in die 1890er-Jahre so, um 1900 waren in Europa immer noch mehr als die Hälfte der Menschen Bauern, derzeit sind es je nach Zählung 2 bis 5\%. Wie war dies möglich? Menschliche und tierische Arbeitskraft wurden durch fossile Energie ersetzt, mit umfassenden Wirkungen auf den ländlichen Raum und die ländliche Gesellschaft (Winiwarter 2013) (vgl. Beitrag 5.1).

Das hatte eine umfassende, auch den menschlichen Körper selbst erfassende Transformation zur Folge. Körpergröße und Lebenserwartung sind gestiegen. Wir können Geburten einleiten und oft den Tod hinauszögern, damit wandeln sich auch Familienstrukturen und soziale Bedürfnisse. Die Menschheit hat, indem sie eine gesellschaft- 
liche Transformation durchgemacht hat, ihr Verhältnis zur inneren und äußeren Natur tiefgreifend gewandelt (Winiwarter 2013). Die Wiener Schule der Sozialen Ökologie hat diese Transformationen mithilfe von langen Zeitreihen des "gesellschaftlichen Stoffwechsels" empirisch fassbar gemacht. Mit Material- und Energieflussanalysen kann sichtbar gemacht werden, dass die fossilenergiebasierte Gesellschaft menschheitsgeschichtlich einmalig ist (vgl. Abbildung 3.4.1). Eine solche Gesellschaft kann nicht stabil sein, weil sie auf fossilen und damit endlichen Ressourcen basiert und weil die Emissionen aus der Nutzung dieser Energiequellen die Aufnahmefähigkeit natürlicher Kohlenstoffsenken wie der Atmosphäre längst übersteigt. Weltweit hat sich seit 1900 die Entnahme von Wasser annähernd verachtfacht, die von Materialien wie Biomasse, Erzen und Mineralien stieg um den Faktor 12 und fast im selben Ausmaß die gesellschaftlich genutzte Primärenergie. Die $\mathrm{CO}_{2}$-Emissionen aus der Verbrennung fossiler Brennstoffe und aus der Zementproduktion stiegen gar um den Faktor 18. Mit Ende der fossilen Ressourcen wird auch diese Dynamik der Ressourcennutzung an ein Ende kommen, und damit wird sich die Gesellschaft selbst tiefgreifend wandeln. Dieser ebenso wünschenswerte wie unvermeidbare Wandlungsprozess wird mit dem Begriff nachhaltige Entwicklung zu fassen und in Programme wie den SDGs umzusetzen versucht.

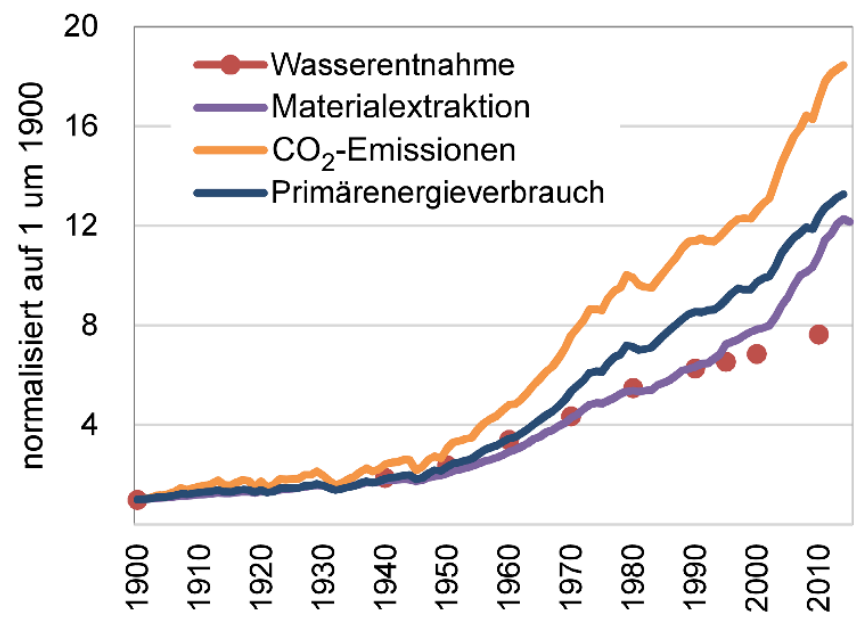

Abbildung 3.4.1: Das 20. Jahrhundert als - fossilenergiegetriebener - Sonderfall der Menschheitsgeschichte (Boden et al. 2017; Krausmann et al. 2009, 2018; Shiklomanov 2000)

Historische Vergleiche erlauben es, heutige Systemzustände besser zu verstehen. Die wesentlichen Treiber des Wandels seit der industriellen Revolution sind neben Bevölkerungswachstum - und der damit einhergehenden Urbanisierung - Technologie und Politik, die ja auch für die Rahmenbedingungen wirtschaftlichen Handelns sorgt. 


\section{Umwelt in Gesellschaft, Politik \& Recht}

Alle diese gesellschaftlichen Prozesse hängen von der Verfügbarkeit von Energie ab (vgl. McNeill 2003).

Seit der industriellen Revolution sind immer wieder kritische Stimmen laut geworden, die davor warnten, die Umwelt weiter zu zerstören. Seit den 1970er-Jahren machte das Wort Umweltschutz Karriere, und Umwelt wurde zur Politikmaterie, nicht nur für "grüne" Parteien, sondern für alle politischen Akteurinnen und Akteure. Seitdem gehört Umweltwissen zur Allgemeinbildung - heute vermutlich mit Schwerpunkt auf Treibhausgase und Klimawandel, früher orientiert an Schmutzskandalen wie dem Dioxinunfall von Seveso, der Reaktorkatastrophe von Tschernobyl oder dem Vogelsterben der 1960er-Jahre durch Dichlordiphenyltrichlorethan, besser bekannt als DDT (Winiwarter 2013). Die Reflexion der Wechselwirkungen zwischen Menschen und Natur ist ein wichtiger Teil des Orientierungswissens für alle Expertinnen und Experten, die in Prozessen nachhaltiger Entwicklung arbeiten.

\subsubsection{Erfolg und Risiko}

Wann immer Menschen in natürliche Systeme absichtsvoll eingreifen (wir nennen dies „kolonisierende Eingriffe“), kommt es, neben den erwünschten oder zumindest vorhergesehenen Folgen, auch zu unbeabsichtigten Wirkungen (Sieferle und MüllerHerold 1996). Mithilfe des Konzepts der „Risikospirale“ wird dieser Zusammenhang fassbar (vgl. Abbildung 3.4.2). Das Wichtigste daran ist die Kombination von Erfolg und Nebenwirkung. Menschen bewältigen ein Risiko erfolgreich. Sie sind dadurch in ihrem Tun bestärkt. Die Nebenwirkungen überraschen sie dann. Wenn Umwelthistorikerinnen und Umwelthistoriker ihre Fallstudien präsentieren, dann ist damit das Ziel verbunden, allen, die heute auf Natur einwirken, vor Augen zu führen, dass Nebenwirkungen typisch und normal sind und keine Ausnahme darstellen. Dies soll zu einer vorsorgenden Innovationskultur beitragen.

Abbildung 3.4.2 veranschaulicht diese Risikospirale am Beispiel der neolithischen Revolution, die u.a. folgende Gesellschafts-Natur-Interaktionen angetrieben haben dürfte: Durch Bevölkerungswachstum und eventuell auch durch Klimaänderung kommt es zu Nahrungsengpässen bei Jägern und Sammlerinnen. (1) Die Menschen werden sesshaft, beginnen den Boden zu bebauen und Vieh zu halten. (2) Dadurch werden sie von der lokalen Witterung abhängig. Speicher sollen Missernten ausgleichen. Die zunehmende Arbeit wird von einer dichteren Bevölkerung geleistet. Speicher sind anfällig für Schädlinge und laden zu Raubzügen ein. (3) Spezialisten für Schädlingsbekämpfung oder den Schutz der Siedlungen müssen zusätzlich ernährt werden. Anbauflächenausweitung kann zu Konflikten führen. (4 \& 5) Wissen wird nötig, um effizient zu produzieren. Ein Teil der Bevölkerung produziert nicht mehr, sondern 


\section{Umwelt in Gesellschaft, Politik \& Recht}

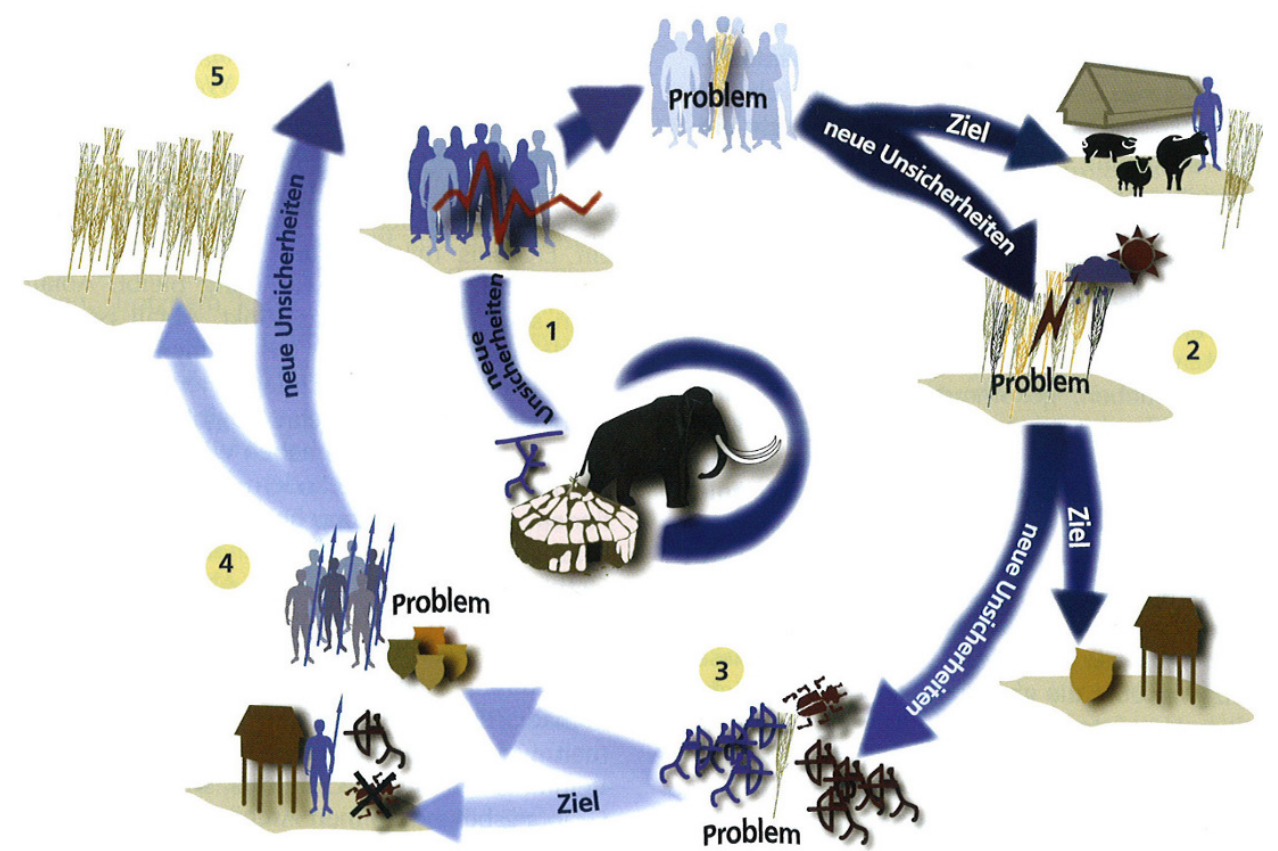

Abbildung 3.4.2: Die Risikospirale am Beispiel der neolithischen Revolution (Winiwarter 2005, S. 147; Graphik: Martin Ober, RGZM)

schreibt und muss von den anderen erhalten werden. Die landwirtschaftliche Produktion muss gesteigert werden, was zu Problemen wie Erosion und Erschöpfung des Bodens, in späteren Jahrhunderten auch zu Überdüngung und Vergiftung des Grundwassers führt. Die Risikospirale dreht sich immer weiter.

Fast ebenso alt ist die Risikospirale der künstlichen Bewässerung. In trockenen Gebieten mit fruchtbaren Böden schien Bewässerung gut für die Nahrungssicherheit zu sein. Bereits im alten Mesopotamien wurden Bewässerungssysteme angelegt. Der Nachteil? Flusswasser, das auf die Felder geleitet wird, enthält viel mehr gelöste Salze als Regenwasser. Spült man den bewässerten Boden nicht regelmäßig, lagert sich mehr und mehr Salz ab. Der Boden wird unfruchtbar. Bis heute sind die Böden in Mesopotamien durch diesen Eingriff vor 5.000 Jahren viel weniger fruchtbar, als sie es zunächst waren. Dieser Effekt wurde bereits vor 60 Jahren beschrieben (Jacobsen und Adams 1958).

Die Ausbeutung überseeischer Kolonien hat das frühneuzeitliche Europa reich gemacht, auch die Niederlande. Infolge der Konfessionskriege des 17. Jahrhunderts wurden sie zur dichtest besiedelten Zone Europas. Fruchtbares Ackerland wurde dringend gebraucht. Eine Strategie, die in dieser feuchten Gegend schon um das Jahr 1000 nachweisbar ist, wurde daher intensiviert. Moore wurden trockengelegt, ein Eingriff, mit dessen Nebenwirkung das Land bis heute kämpft. Torfmoore bestehen aus nur wenig 


\section{Umwelt in Gesellschaft, Politik \& Recht}

zersetztem Pflanzenmaterial, das unter Luftabschluss erhalten bleibt, solange der Moorboden mit Wasser gefüllt ist. Leitet man das Wasser ab, zersetzen auf Sauerstoff angewiesene Mikroorganismen die Pflanzenreste. Dadurch wurde der Boden fruchtbarer. Das hatte aber auch Nebenwirkungen, weil das zersetzte Material viel weniger Platz braucht. Die trockengelegten Torfmoore sanken in sich zusammen, das Land sackte ab und war zunehmend von Überflutung bedroht (TeBrake 2002; Van Dam 2001).

Diese Nebenwirkung führte zur nächsten Intervention. Die Niederländer bauten Deiche, um das neue Ackerland vor Sturmfluten zu schützen. Deichgenossenschaften warteten diese Infrastrukturen. Das klappte hervorragend und beförderte wohl auch die Demokratie im Land. Doch die Absenkung ging hinter den Deichen weiter. In vielen Gegenden sank das Land bis zum Grundwasserspiegel. Wollte man eine sekundäre Vernässung verhindern, musste das Wasser abgepumpt werden, um Ackerland und Weiden zu erhalten. Auch hier erwiesen sich die Niederländer als kreativ im Umgang mit den Nebenwirkungen ihrer Innovationen. Die Windmühlen, für die Holland berühmt ist, pumpen das Wasser ab. Pumpt man aber das Süßwasser in einem küstennahen Gebiet ständig ab, dringt irgendwann Salzwasser über den Untergrund ein. In den Niederlanden ist das an einigen Stellen bereits passiert. Die ersten Entwässerungsgräben wurden um 1200 angelegt. Seit mehr als 800 Jahren ist man also in den Niederlanden mit der Beherrschung der Nebenwirkungen eines einzigen Eingriffs, der Trockenlegung der Moore, beschäftigt (TeBrake 2002; Van Dam 2001).

Solche „Risikospiralen“ findet man an vielen Orten und in vielen Zusammenhängen. Flussregulierungen schützen einen Ort vor Hochwasser, weil sie es woandershin verlagern. Staudämme verändern nicht nur den Grundwasserspiegel, anders als man gedacht hätte, sondern auch den Feststofftransport in den Flüssen. Nach den Ölpreisschocks 1973 und 1979 sahen viele Regierungen Kernkraftwerke als eine Möglichkeit, die gefährliche Abhängigkeit von fossilen Brennstoffen zu vermindern. Allerdings ist bis heute das Problem der Endlagerung radioaktiver Abfälle aus solchen Kraftwerken ungelöst (Winiwarter 2013).

Die Zeit der industriellen Revolution hat Umwelthistorikerinnen und -historiker stark beschäftigt, weil hier die Nebenwirkungen menschlicher Handlungen auf die Natur besonders auffällig wurden. Die heutige industrielle Lebensweise ist durch Güter gekennzeichnet, bei deren Herstellung und Gebrauch es zur Verschmutzung von Boden, Wasser und Luft kommt. Während der ersten Phase der Industrialisierung wurden die Produktionszentren verschmutzt. Das Wort Smog (smoke \& fog) wurde zur Beschreibung der dicken Londoner Luft kreiert, die durch Abgase von Produktionsbetrieben entstand (Brimblecombe 1987). Seit der Transformation zur Konsumgesellschaft in den 1950er-Jahren führt nicht nur die Produktion, sondern auch der 
Gebrauch von Gütern zu weit gestreuter Verschmutzung. Auch diese Entwicklung lässt sich als Risikospirale erzählen (Winiwarter 2013). Doch es geht nicht nur um Güter.

In den Industriestaaten dominiert der tertiäre Sektor die Ökonomie. Die Wertschöpfung stammt zu einem Gutteil aus Dienstleistungen. Im Tourismusland Österreich drehen sich auch dienstleistungsgetriebene Risikospiralen, allen voran jene des Wintertourismus. Sie wurde zu Beginn der 1950er-Jahre, als fossile Energie im Verhältnis $\mathrm{zu}$ anderen Gütern relativ billiger wurde, durch das größte Wiederaufbauprogramm der Geschichte, den Marshallplan, in Gang gesetzt. Österreich förderte damit im Einklang mit dem Geberland USA den Bau von Skiliften und anderen Wintersporteinrichtungen. Billige Kredite lockten Investorinnen und Investoren, die die Herausforderungen der alpinen Natur oft unterschätzten. Bau- und Erhaltungskosten überstiegen die geplanten Summen deutlich. In den 1970er-Jahren gab es in Österreich keinen einzigen Skiliftbetrieb, der schwarze Zahlen geschrieben hätte. Viele waren stark verschuldet. Schon des Zinsendienstes wegen mussten sie jährlich ihre Umsätze und Gewinne steigern. Wachstum war alternativlos, aber nur möglich auf Kosten anderer Destinationen. Ein erbitterter Konkurrenzkampf führte zunächst v.a. zur Steigerung der Geschwindigkeiten und der Transportkapazitäten der Lifte. Die Konkurrenzfähigkeit wurde mit massiven Investitionen erkauft. Die Schuldenspirale drehte sich weiter. Die Pisten wurden voller und voller, weil mehr Personen je Zeiteinheit an den Bergstationen ausstiegen. Eine Ausweitung der Pistenflächen war nur begrenzt möglich, aus naturräumlichen wie gesellschaftlichen Gründen. Also ging man an die Ausweitung der Zeit, in der die Pisten befahrbar waren. Der Rasenskilauf im Sommer scheiterte schnell. Flutlichtanlagen machten die Nacht zum Tag, waren aber nur beschränkt ausbaufähig. Pistenraupen stellten problemlos schnell befahrbare Oberflächen her. Das Schneemanagement wurde von Jahr zu Jahr perfekter. Die Schneekanone machte die Wintersportorte unabhängiger vom Wetter, denn Schneemangel konnte ein Gebiet voller teurer, fremdfinanzierter Infrastruktur schnell in den Ruin treiben. Schnee wurde zur teuren Ware. Um mit ihr sparsamer umzugehen, wurden die Pisten von Senken und Hügeln befreit, um den Preis höherer Erosion im Sommer. Die Pistenbegrünung ist inzwischen entsprechend perfektioniert, mit Folgen für lokale Tier- und Pflanzenwelten. Kunstschnee braucht Wasser, das im Sommer in Speicherteichen gesammelt wird und daher der lokalen Landschaft entzogen wird - mit unabsehbaren Langzeitfolgen für die Ökosysteme, die gerade in den Alpen durch den Klimawandel bereits unter Stress stehen. In den teuersten Skiorten werden inzwischen Kühlschlangen im Boden verlegt, um die kostbare weiße Pracht möglichst lange zu erhalten. Jeder Schritt, vom Ausbau der Skilifte, um der Abwanderung und der Armut zu begegnen, bis hin zur Piste mit Outdoorkühlschrank war eine erfolgreiche Intervention in natürliche Systeme. Jeder Schritt hatte Nebenwirkungen, die wiederum Eingriffe erforderten. Ein Ende ist nicht abzusehen. Vom 


\section{Umwelt in Gesellschaft, Politik \& Recht}

Carver, der enge Schwünge platzsparend erlaubt und damit Pisten entlastet, zur Verwendung von Bakterien als Kristallisationskeime für sparsamere Schneeherstellung, die dann auch bei Temperaturen über dem Gefrierpunkt möglich wird, bis zur GPS-präzisionsgesteuerten Pistenraupe reichen die Intensivierungsbemühungen der letzten Jahre. Mit weiteren Nebenwirkungen ist zu rechnen (Groß 2019).

\subsubsection{Lernen von vorindustriellen Ökonomien}

Der britische Wirtschaftshistoriker E. A. Wrigley (1988) hat die vorindustrielle Welt eine wood economy, eine Holzwirtschaft, genannt. Alle gesellschaftlichen Bereiche, so auch die Kriegsführung, waren auf Land angewiesen. Daher lassen sich Dynamiken einer auf nachwachsenden Rohstoffen basierenden Ökonomie an der Geschichte gut studieren (siehe Fallbeispiel 3.4.1).

\section{Fallbeispiel 3.4.1: Venedig und die Eichen (Appuhn 2009)}

Kriegs- wie Handelsschiffe wurden aus Eichenholz gebaut, weil es hart und tanninreich und gegen Meerwasser und Fäulnis daher weit beständiger ist als andere Holzarten. Allerdings wachsen Eichen langsam. Mächtige Stadtstaaten an den Küsten des heutigen Italien kämpften jahrhundertelang um die Vorherrschaft im Mittelmeerraum. Amalfi, Pisa, Genua und Venedig betrieben Flottenbauwerkstätten, die große Mengen Eichenholz benötigten. Im Jahr 1476 erließ der venezianische Senat sechs Regeln für die Nutzung seiner kommunalen Wälder auf der terra ferma, dem zu Venedig gehörenden Festland. Um die Versorgung mit den unverzichtbaren Eichen zu sichern, verbot der Senat die Waldweide, die Ernte von Feuerholz und das Legen von Feuern, um das Unterholz zu entfernen. Mehr als zweihundert Jahre später und nach weiteren Verschärfungen der Regulierung - sogar das Sammeln von Totholz wurde schließlich verboten - kamen die Beamten der Signoria zum Schluss, dass nicht mehr, sondern weniger Eichen wuchsen. Sie beschuldigten die Bauern, die Wälder entgegen den Verboten doch genutzt zu haben. Das Gegenteil war der Fall. Solange die Bauern bei der Nutzung des Waldes als Weide oder zur Feuerholzsammlung Unterholz und Bäume von geringerer Qualität entfernt hatten, gab es mehr Platz für einzelne Eichen, die dann zu den gesuchten großen Bäumen heranwachsen konnten. Hielten sie sich an die Verbote, wuchsen weniger Eichen.

Doch auch die Venezianer selbst spielten für den Eichenwald eine Rolle, die sie nicht erkannten. Solange die Schiffsbauer genug Eichen verbrauchten, war der Eichenertrag noch einigermaßen akzeptabel; sobald der Bedarf nachließ, verblieben Unterholz und dünne Stämme, die bei der Eichenernte mit entfernt wurden, im Wald und erstickten den Nachwuchs junger Eichen, die zu Bäumen für den Schiffsbau hätten heranwachsen können. Je sparsamer die Venezianer waren, umso schlechter sah es mit dem Nachwuchs aus.

Ohne die Ökologie des Eichenwaldes zu berücksichtigen, kann die Entwicklung der Erträge aus den venezianischen Wäldern nicht verstanden werden. Das Ökosystem Wald spielt eine zentrale Rolle. Die Bedingungen, unter denen Eichen besonders gut oder besonders schlecht wachsen, waren den Beamten in der Zentrale nicht bekannt. Die Ansichten der lokalen Bevölkerung, die sehr wohl verstand, dass Eichenwald einer regelmäßigen Entnahme von konkurrierenden Arten bedurfte, sogar die schriftlichen Vorschläge jener Beamten, die mit dem Eichenwald vertraut waren und der Signoria gut durchdachte Pläne vorlegten, wie durch mehr Entnahme von Feuerholz der Mangel daran behoben und das Eichenwachstum befördert werden könnte, wurden allesamt abgetan. 
Die im Fallbeispiel 3.4.1 geschilderten venezianischen Ordnungsversuche des 15. Jahrhunderts lassen sich gut in Beziehung setzen zu aktuellen Fragen wie der Herstellung von Kohlenstoffsenken durch Aufforstung zur Erreichung der Kyoto-Ziele (Winiwarter 2013) oder den Visionen, Afrika mit Wald zu überziehen. Lokales Wissen wird häufig abgewertet, während in den Machzentralen weitab vom Geschehen auf glänzendes Papier gedruckte Pläne vorliegen, die lokale - ökologische wie soziale - Verhältnisse ignorieren und schon deshalb scheitern müssen. Oft liegt ihnen eine technokratische Vision zugrunde, die die Steuerbarkeit und Veränderbarkeit von Natur überschätzt, die Komplexität im Zweifel eher unterschätzt. Das Orientierungswissen der Umweltgeschichte ist hier korrigierend und daher besonders kostbar.

\subsubsection{Wahrnehmung wandelt sich}

Während man 1940 mit einem Futterhäuschen namens „Kontraspatz“ noch Sperlinge von der Winterfütterung auszuschließen suchte, wurde der Hausspatz 2002 zum „Vogel des Jahres“. Während zwischen 1940 und 1945 in Berlin die radioaktive Zahncreme Doramad produziert wurde, die strahlend weiße Zähne und Bakterienabtötung versprach, untersucht man heute besorgt die Effekte geringer radioaktiver Belastungen. Während heute viele Menschen bei geöffnetem Fenster schlafen, plagte den späteren amerikanischen Präsidenten John Adams 1776 die Vorstellung, dass Nachtluft gefährlich sei, und er bestand darauf, nur bei geschlossenem Fenster zu schlafen (Baldwin 2003). Während früher Kurorte mit ozonreicher Luft warben, gibt es heute Warnsysteme, die gefährdete Personen davon abhalten sollen, bei hoher Ozonkonzentration aus dem Haus zu gehen (Winiwarter 2013).

Geänderte Wirtschaftsweisen geben oft den Ausschlag für einen Bewertungswandel. Im frühneuzeitlichen England war der Misthaufen Teil der Verlassenschaftsabhandlung einer Person. Die Nährstoffe wurden in Geld bewertet (King 1992). In manchen Gegenden Asiens lassen sich Bäuerinnen und Bauern bis heute stolz vor ihrem Misthaufen photographieren - ein großer Misthaufen steht für Reichtum, weil Nährstoffe in der Landwirtschaft entscheidend sind. Mit der Verfügbarkeit von synthetischem Dünger und der räumlichen Segregation von Viehzucht und Ackerbau wurde in den Industrieländern ein Bewertungswandel eingeleitet. Gülle ist heute ein Umweltproblem, kein Aktivposten einer Verlassenschaft (Winiwarter 2013).

Die Wahrnehmung von Naturphänomenen ist nicht ohne Grund einem Wandel unterworfen. Mal beeinflussen geänderte Vorstellungen der Gesundheit und des menschlichen Körpers die Wahrnehmung, mal liegt es - wie im Fall der Nachtluft am Zusammenwirken von technischen Innovationen, geänderten Lebensgewohnheiten und an einer neuen Theorie der Ansteckung. Wenn Umwelthistorikerinnen und 


\section{Umwelt in Gesellschaft, Politik \& Recht}

-historiker dem Wahrnehmungswandel nachgehen, erforschen sie Zusammenhänge zwischen Technik, Wirtschaft, Wissenschaft und Natur (Winiwarter 2013).

Umweltgeschichte zeigt den Wandel von Bewertungen. Sehr wahrscheinlich werden sich auch heutige Bewertungen ändern. Was aktuell als gut gilt, mag in einem Jahrzehnt schockierend sein. Was wir heute für schlecht halten, könnte künftig positiv bewertet werden. Wir sollten also nicht allzu sicher sein, recht zu haben (Winiwarter 2013). Darin liegt eine Leistung umwelthistorischer Arbeit: Sie ruft zur Bescheidenheit auf, zu einem Auftreten als Expertin oder Experte, der/dem die prinzipielle Unsicherheit und Vorläufigkeit selbst der besten wissenschaftlichen Studie bewusst ist.

\subsubsection{Späte Lehren aus frühen Warnungen}

Die Europäische Umweltagentur (EEA) veröffentlichte in den Jahren 2001 und 2013 zwei bemerkenswerte Berichte. Darin werden Fälle von Umweltverschmutzung und anderen Umweltschäden vorgestellt, bei denen aus sehr frühen Warnungen erst sehr spät Lehren gezogen wurden. Die Beispiele reichen von DDT über Quecksilber bis hin zu Tabak und Beryllium, das in der Rüstungsindustrie eine wichtige Rolle spielt. Eines sei kurz herausgegriffen, das feuerfeste Wundermaterial Asbest (siehe Fallbeispiel 3.4.2).

\section{Fallbeispiel 3.4.2: Asbest als Beispiel für späte Lehren aus frühen Warnungen (EEA 2001)}

Feuer war eine der häufigsten Todesursachen in einer zunehmend urbanisierten Welt, in der immer mehr Menschen in brennbaren Gebäuden lebten und Zeit in öffentlichen Einrichtungen verbrachten. Der Großbrand von 1903 in Chicago, bei dem im Iroquois Theatre 602 Besucher verbrannten, Feuer in Hotels, Textilfabriken, Schulen und Gefängnissen mit jeweils mehreren Hundert Toten erforderten eine gesellschaftliche Reaktion. Eine Lösung fand man in der Technologie: Das feuerfeste Material Asbest wurde zur Standardlösung. Wie so viele technische Entwicklungen wurde auch die Verwendung von Asbest im Zweiten Weltkrieg beschleunigt. Der Brandschutz von Schiffen war ein Schwerpunkt im Schiffbau. Das amerikanische Verteidigungsministerium stufte Ende 1939 die Mineralfaser als "kritisches Material“ ein. Gesundheitliche Probleme traten zuerst in den Asbestfabriken auf, die englische Arbeiterin Nellie Kershaw (1891-1924) erlangte traurige Berühmtheit als erstes durch Autopsie bestätigtes Opfer der Lungenkrankheit Asbestose. Die Untersuchung ihres Falles führte erst in den 1930er-Jahren zu arbeitsrechtlichen Regelungen, doch hatte es bereits in den 1890er-Jahren Warnungen gegeben. Die Fabrikinspektorin Lucy Deane Streatfeild war eine der Ersten, die die Symptome genau beschrieb, mit der Härte und Struktur der feinen Fasern in Beziehung setzte und damit im Grunde den heutigen Wissensstand vorwegnahm. Ihre Warnungen verhallten ungehört. Auch das französische Gewerbeaufsichtsamt meldete bereits Anfang des 20. Jahrhunderts 50 Todesfälle durch Lungenerkrankungen bei weiblichen Asbest-Textilarbeitern (Auribault 1906). Doch Asbest wurde und wird weiterhin abgebaut und verwendet. Zwar haben mehr als 50 Länder, darunter das Vereinigte Königreich, Australien und alle Mitgliedsstaaten der Europäischen Union, die Verwendung von Asbest verboten, aber die USA importieren und verwenden weiterhin Asbest, ohne Pläne für strengere Vorschriften zu haben. Asbestprobleme treten immer dann auf, wenn verbauter Asbest an die Oberfläche gelangt. Der mit Asbest hergestellte Eternit ist nach wie vor auf Dächern zu finden, in Bremsbelägen, Kupplungen, 
Vinylfliesen und einigen Zementrohren. Dies, obwohl völlig zweifelsfrei feststeht, dass Asbest genotoxisch und krebserregend ist, dass eine Krebsart, das Pleuramesotheliom, ausschließlich durch Asbest entsteht. Wieso ist es möglich, ein so gefährliches Material weiterhin abzubauen und zu verwenden? Das liegt an den langen Latenzzeiten. Asbestose wird erst nach $10-30$ Jahren symptomatisch. Das Mesotheliom tritt erst nach etwa 40 Jahren auf. Das heißt, dass die gesundheitlichen Schäden durch Asbest in den nächsten Jahrzehnten ansteigen werden. Ein Asbestverbot in den Niederlanden schon im Jahre 1965 statt erst 1993 hätte 34.000 Todesfälle verhindert.

Gerade für Umweltbelastungen, die sich erst nach langer Latenzzeit bemerkbar machen, ist das Lernen aus frühen Erfahrungen entscheidend. Darin liegt ein konkreter Beitrag der Umweltgeschichte zu SDG 3 (Gesundheit), aber auch SDG 12 (verantwortliche Produktion) und SDG 5 (Gendergerechtigkeit) sind angesprochen.

\subsubsection{Altlasten und Ewigkeitskosten}

Wer das Wort „Altlasten“ hört, denkt vielleicht an Endlager für abgebrannte Brennstäbe von Atomkraftwerken. Doch der Begriff umfasst alle Überreste von industriellen Aktivitäten, die im Boden verblieben sind und eine mögliche Gefahr etwa für das Grundwasser darstellen. Besonders aufwendig ist der Umgang mit Bergbauvermächtnissen. Eine große Altlast ist der ehemalige Kohlebergbau im dicht besiedelten deutschen Ruhrgebiet. Fast die Hälfte des Ruhrgebiets liegt heute etliche Meter unter dem Grundwasserspiegel. Mehr als 100 Pumpwerke müssen dauerhaft laufen. Das Wasser wird in die eingedeichte Emscher gepumpt. Solange das Ruhrgebiet bewohnbar bleiben soll, ist das Abpumpen großer Wassermengen und die Sicherung von Stollenanlagen auf „ewig“ nötig. Dafür sind zu derzeitigen Kostensätzen 220 Mio. Euro pro Jahr als „Ewigkeitskosten“ zu budgetieren (RAG 2016). Doch das ist nur eine der vielen Altlasten, die die Optionen künftiger Generationen einschränken.

Die meisten Altlasten entstanden ab der Industrialisierung des 19. Jahrhunderts, vermehrt seit der "Großen Beschleunigung" (Great Acceleration) nach dem Zweiten Weltkrieg mit dem Umstieg auf Erdöl und Erdgas als Schlüsselenergieträger. Das dafür nötige globale Energieversorgungssystem ist das größte jemals errichtete technische Infrastrukturnetzwerk (Seto et al. 2016). Es überzieht die gesamte Erde, umfasst u.a. Förderstellen offshore und onshore, Pipelines, Raffinerien, Kraftwerke und Tankstellen. All das sind (potenzielle) Altlasten, die auf lange Zeit überwacht und gesichert werden müssen. Viele nukleare Altlasten gehen auf das Wettrüsten im „Kalten Krieg“ zwischen den damaligen Supermächten USA und UdSSR zurück. Uranabbaustätten, Atomwaffenfabriken und Bombenversuchsgelände sind über die ganze Welt verstreut, in den Wüsten Nordamerikas ebenso wie in den Steppen Zentralasiens oder auf Inseln im Pazifik. Dazu kommen deutlich ältere Altlasten v.a. aus dem Bergbau, die oft durch europäischen Kolonialismus entstanden. In Huancavelica in Peru etwa beutete die 


\section{Umwelt in Gesellschaft, Politik \& Recht}

spanische Kolonialmacht in der frühen Neuzeit Menschen und Natur aus, um an das Quecksilber zu kommen, das sie für die Silberproduktion brauchte. Heute, fast fünfhundert Jahre später, ist Huancavelica eines der am stärksten mit Quecksilber verseuchten städtischen Gebiete der Welt, mit entsprechend negativen gesundheitlichen Folgen für die lokale Bevölkerung (Winiwarter und Bork 2014, S. 78f.).

Altlasten aus der Vergangenheit, ob aus der jungen oder ferneren, schränken die Möglichkeiten nachhaltiger Entwicklung ein, bekommen aber wenig Aufmerksamkeit in der aktuellen Debatte. Kein SDG adressiert sie gezielt. Umweltgeschichte bringt sie zurück ins Bewusstsein und macht darauf aufmerksam, dass auch heutige Entscheidungen noch Zehntausende Jahre oder länger - man denke etwa an die Klimakatastrophe oder die Versauerung der Ozeane - Auswirkungen haben werden.

\subsubsection{Schlussbemerkung}

Umweltgeschichte ermöglicht Lernen aus Distanz. Indem sie den historischen Wandel von Wahrnehmung analysiert, relativiert sie zugleich den Wahrheitsanspruch heutiger Wahrnehmungen. Verantwortungsvolle Expertise - auch im Umwelt- und Nachhaltigkeitsbereich - beruht auf der Einsicht, dass jedes Wissen prinzipiell unsicher, unvollständig und vorläufig ist. Umweltgeschichte trägt damit zur Entwicklung einer wichtigen Kompetenz in einer Gesellschaft bei, die sich mit einer Flut von Informationen ebenso konfrontiert sieht wie mit fake news und alternative facts. Diese wichtige Kompetenz heißt Informationskritik.

Umweltgeschichte macht die langfristigen und nebenwirkungsreichen Folgen gesellschaftlicher Eingriffe in Ökosysteme sichtbar. Wie am Eichenwald der Venezianer zu sehen, kann Ressourcennutzung nur dann langfristig erfolgreich sein, wenn sie auf einem umfassenden Verständnis der Eigendynamik der Ökosysteme basiert. Umweltgeschichte erinnert an Altlasten und Ewigkeitskosten als wesentliche, zu wenig beachtete Rahmenbedingungen nachhaltiger Entwicklung. Sie zeigt, dass auch erfolgreiche Lösungen von Nachhaltigkeitsproblemen in der Vergangenheit immer mit Nebenwirkungen einhergingen, die wieder Handlungsdruck erzeugten („Risikospiralen“). Wer das verstanden hat, denkt anders über das Verhältnis zwischen Innovation und Vorsorgeprinzip nach.

Indem Umweltgeschichte als historische Ausprägung der Sozialen Ökologie die Entwicklung und die Muster des Energie- und Materialverbrauchs untersucht, macht sie klar, in welch menschheitsgeschichtlich außerordentlicher und instabiler Situation hochentwickelte Gesellschaften, wie die unsere nach der Industrialisierung des 19. und 20. Jahrhunderts, stecken (Haberl et al. 2016). Energiesysteme sind eine folgen- 
reiche Facette des Umgangs einer Gesellschaft mit Natur. Der Wandel des Energiesystems zu erneuerbaren Energieträgern wird, das lehrt die Geschichte vergangener Energietransitionen, mit einem umfassenden gesellschaftlichen Wandel - einer sozialökologischen Transformation - einhergehen.

Die Umwelteffekte menschlicher Eingriffe treten manchmal schleichend und mitunter sehr schnell ein. Späte Lehren aus frühen Warnungen, wie sie etwa in der Geschichte der Nutzung von Asbest sichtbar wurden, zeigen, wie lange gesellschaftliche Lernprozesse dauern können. Die Folgen menschlicher Eingriffe in die Natur sind in allen Umweltsystemen - Boden, Wasser, Luft und Landschaft - präsent, und alle Lebewesen können davon betroffen sein. Wir vermögen ausgestorbene Tiere nicht wieder lebendig zu machen, aber wir können verhindern, dass weitere aussterben. Wir vermögen die Natur früherer Zeiten nicht wiederherzustellen, können aber für ihre und damit unsere Zukunft Vorsorge tragen. Dafür müssen wir über frühere Nutzungen und den Zustand vergangener Umwelten Bescheid wissen. Umweltgeschichte ist eine Wissenschaft von der Vergangenheit, aber für die Zukunft (Winiwarter 2013).

\section{Literatur}

Auribault, M. (1906): Note sur l'hygiène et la sécurité des ouvriers dans les filateurs et tissages d'amiante. Bulletin d'Inspection du Travaille, 14, 120-132.

Appuhn, K. (2009): A Forest on the Sea: Environmental Expertise in Renaissance Venice. Baltimore: Johns Hopkins University Press.

Baldwin, P. C. (2003): How night air became good air 1776-1930. Environmental History, 8, 3, 412-429. https://doi.org/10.2307/3986202.

Boden, T. A., Marland, G., and Andres, R. J. (2017): Global, Regional, and National Fossil-Fuel CO2 Emissions. Carbon Dioxide Information Analysis Center (CDIAC). Oak Ridge, Tenn., USA: Oak Ridge National Laboratory, U.S. Department of Energy. https://doi.org/10.3334/CDIAC/00001 V2013.

Brimblecombe, P. (1987): The Big Smoke. A History of Air Pollution in London since Medieval Times. London: Routledge.

EEA (European Environment Agency) (2013): Late Lessons from Early Warnings: Science, Precaution, Innovation. Luxembourg: Publications Office of the European Union. Available at: https://www.eea.europa.eu/publications/late-lessons-2 [accessed 11.3.2019].

Groß, R. (2019): Die Beschleunigung der Berge. Eine Umweltgeschichte des Wintertourismus in Vorarlberg/Österreich (1920-2010). Köln, Weimar, Wien: Böhlau.

Haberl, H., Krausmann, F., Fischer-Kowalski, M., and Winiwarter, V. (eds.) (2016): Social Ecology. Society-Nature Relations across Time and Space. Springer.

Jacobsen, T. and Adams, R. M. (1958): Salt and silt in ancient Mesopotamian agriculture: Progressive changes in soil salinity and sedimentation contributed to the breakup pf past civilizations. Science, 128, 3335, 1251-1258. https://doi.org/10.1126/science.128.3334.1251.

King, W. (1992): How high is too high? Disposing of dung in seventeenth-century prescot. Sixteenth Century Journal, 23, 3, 443-457. https://doi.org/10.2307/2542488.

Krausmann, F., Gingrich, S., Eisenmenger, N., Erb, K. H., Haberl, H., and Fischer-Kowalski, M. (2009): Growth in global materials use, GDP and population during the 20th century. Ecological Economics, 68, 2696-2705. https://doi.org/10.1016/j.ecolecon.2009.05.007. 


\section{Umwelt in Gesellschaft, Politik \& Recht}

Krausmann, F., Lauk, C., Haas, W., and Wiedenhofer, D. (2018): From resource extraction to outflows of wastes and emissions: The socioeconomic metabolism of the global economy, 1900-2015. Global Environmental Change, 52, 131-140. https://doi.org/10.1016/i.gloenvcha.2018.07.0 03.

McNeill, J. R. (2003): Blue Planet: Die Geschichte der Umwelt im 20. Jahrhundert. Frankfurt a. M.: Campus.

RAG (Ruhrkohle Aktiengesellschaft) (2016): Aufgaben für die Ewigkeit: Grubenwasserhaltung, Poldermaßnahmen und Grundwassermanagement im Ruhrgebiet. Verfügbar in: https://www.rag.de/verantwortung/handlungsfelder/ewigkeitsaufgaben/ [Abfrage am 11.3.2019].

Seto, K. C., Davis, S. J., Mitchell R. B., Stokes, E. C., Unruh, G., and Ürge-Vorsatz, D. (2016): Carbon lockin: Types, causes, and policy implications. Annual Review of Environment and Resources, 41, 425-452. https://doi.org/10.1146/annurev-environ-110615-085934.

Shiklomanov, I. A. (2000): Appraisal and assessment of world water resources. Water International, 25, 1, 11-32. https://doi.org/10.1080/02508060008686794.

Sieferle, R. P. und Müller-Herold, U. (1996): Überfluß und Überleben: Risiko, Ruin und Luxus in primitiven Gesellschaften. GAIA Ecological Perspectives for Science and Society, 5, 3-4, 135-143. https://doi.org/10.14512/gaia.5.3-4.5.

TeBrake, W. H. (2002): Taming the waterwolf. Hydraulic engineering and water management in the Netherlands during the Middle Ages. Technology and Culture, 43, 3, 475-499. https://www.jstor.org/stable/25147956.

Van Dam, P. J. E. M. (2001): Sinking peat bogs. Environmental change in Holland, 1350-1550. Environmental History, 6, 1, 32-45. https://www.jstor.org/stable/3985230.

WBGU (Wissenschaftlicher Beirat der Bundesregierung Globale Umweltfragen) (2011): Factsheet Nr. 4: Transformation zur Nachhaltigkeit. Verfügbar in: https://www.wbgu.de/factsheets/factsheet42011/ [Abfrage am 11.3.2019].

WCED (World Commission on Environment and Development) (1987): Our Common Future. Oxford: Oxford University Press.

Winiwarter, V. (2005): Niemand ist eine Insel - einführende Bemerkungen zur Umweltgeschichte. In: Daim, F. und Neubauer, W., Hrsg., Zeitreise Heldenberg - Geheimnisvolle Kreisgräben. Niederösterreichische Landesausstellung 2005 (= Katalog des Niederösterreichischen Landesmuseums, Neue Folge Nr. 459). Horn, Wien: Verlag Berger, 146-149.

Winiwarter, V. (2013): Umweltgeschichte: eine Einführung. In: Beier, R., Ecker, A., Edel, K., Ennagi, A., Paireder, B. und Suschnig. H.-M., Hrsg., Umweltgeschichte. historisch-politische bildung. Themendossiers zur Didaktik von Geschichte, Sozialkunde und Politischer Bildung. N ${ }^{\circ} 5$. Wien: Fachdidaktikzentrum für Geschichte, Sozialkunde und Politische Bildung der Universität Wien, 9-16. Verfügbar in: https://geschichte.uni-graz.at/de/geschichtsdidaktik/publikationen/ [Abfrage am 20.7.2019].

Winiwarter, V. und Bork, H.-R. (2014): Geschichte unserer Umwelt. Sechzig Reisen durch die Zeit. Darmstadt: Primus.

Winiwarter, V. und Knoll, M. (2007): Umweltgeschichte: Eine Einführung. Wien, Köln, Weimar: Böhlau UTB.

Wrigley, E. A. (1988): Continuity, Chance and Change. The Character of the Industrial Revolution. Cambridge: Cambridge University Press.

\footnotetext{
OpenAccess Dieses Kapitel wird unter der Creative Commons Namensnennung - Nicht kommerziell 4.0 International Lizenz (http://creativecommons.org/licenses/by-nc/4.0/deed.de)veröffentlicht, welche die nicht-kommerzielle Nutzung, Vervielfältigung, Bearbeitung, Verbreitung und Wiedergabe in jeglichem Medium und Format erlaubt, sofern Sie den/die ursprünglichen Autor(en) und die Quelle ordnungsgemäß nennen, einen Link zur Creative Commons Lizenz beifügen und angeben, ob Änderungen vorgenommen wurden.

Die in diesem Kapitel enthaltenen Bilder und sonstiges Drittmaterial unterliegen ebenfalls der genannten Creative Commons Lizenz, sofern sich aus der Abbildungslegende nichts anderes ergibt. Sofern das betreffende Material nicht unter der genannten Creative Commons Lizenz steht und die betreffende Handlung nicht nach gesetzlichen Vorschriften erlaubt ist, ist auch für die oben aufgeführten nicht-kommerziellen Weiterverwendungen des Materials die Einwilligung des jeweiligen Rechteinhabers einzuholen.
}

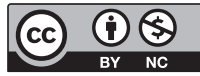

